\title{
Extended I-Love relations for slowly rotating neutron stars
}

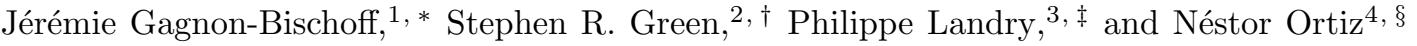 \\ ${ }^{1}$ Department of Physics, University of Ottawa, 75 Laurier Ave E, Ottawa, Ontario, K1N 6N5, Canada \\ ${ }^{2}$ Max Planck Institute for Gravitational Physics (Albert Einstein Institute), Am Mühlenberg 1, 14476 Potsdam, Germany \\ ${ }^{3}$ Enrico Fermi Institute and Kavli Institute for Cosmological Physics, \\ The University of Chicago, 5640 South Ellis Avenue, Chicago, Illinois, 60637, USA \\ ${ }^{4}$ Perimeter Institute for Theoretical Physics, 31 Caroline Street North, Waterloo, Ontario, N2L 2Y5, Canada
}

(Dated: April 3, 2018)

\begin{abstract}
Observations of gravitational waves from inspiralling neutron star binaries - such as GW170817can be used to constrain the nuclear equation of state by placing bounds on stellar tidal deformability. For slowly rotating neutron stars, the response to a weak quadrupolar tidal field is characterized by four internal-structure-dependent constants called "Love numbers". The tidal Love numbers $k_{2}^{\mathrm{el}}$ and $k_{2}^{\mathrm{mag}}$ measure the tides raised by the gravitoelectric and gravitomagnetic components of the applied field, and the rotational-tidal Love numbers $\mathfrak{f}^{\circ}$ and $\mathfrak{k}^{\circ}$ measure those raised by couplings between the applied field and the neutron star spin. In this work we compute these four Love numbers for perfect fluid neutron stars with realistic equations of state. We discover (nearly) equation-of-state independent relations between the rotational-tidal Love numbers and the moment of inertia, thereby extending the scope of I-Love-Q universality. We find that similar relations hold among the tidal and rotational-tidal Love numbers. These relations extend the applications of I-Love universality in gravitational-wave astronomy. As our findings differ from those reported in the literature, we derive general formulas for the rotational-tidal Love numbers in post-Newtonian theory and confirm numerically that they agree with our general-relativistic computations in the weak-field limit.
\end{abstract}

\section{INTRODUCTION}

The Advanced LIGO and Virgo gravitational-wave observatories have recently detected the inspiral and merger of two low-mass compact objects consistent with neutron stars (NSs) [1. With three operating detectors and the simultaneous observation of a gamma-ray burst, it was possible to localize the event in the sky and perform follow-up observations of electromagnetic counterparts. Together, these exciting observations have provided a host of insights into neutron star physics, gamma-ray bursts, kilonovae, and even cosmology.

The inspiral stage of the binary NS merger, seen only by the gravitational wave detectors, can provide insight into the internal structure and composition of the NSs through their tidal deformability. As the stars inspiral, they exert tidal forces on each other, resulting in deformed stars. This affects the orbital dynamics of the binary, and slightly accelerates the coalescence 2 4. Indeed, through nonobservation of an unambiguous tidal phase shift, Ref. 1] placed bounds on the tidal deformability of the stars and thereby constrained competing nuclear physics models of the NS interior.

Cold NS matter is described as a barotropic perfect fluid with a particular equation of state (EoS) determined by the underlying nuclear physics model. In recent years, much effort has been devoted to describing the tidal deformability of compact objects in general relativity, including NSs, as a function of their EoS [5-10]. The main result of this program is that for weak and slowly varying tides the dependence on the EoS is captured by a set of constants called Love numbers. The Love numbers relate the induced field of the tidally deformed body to the applied tidal field.

The analysis of Ref. [1] constrained only the leading-order tides raised by the presence of the binary companion, but as detector sensitivity is improved it will become important to have waveform predictions that also take into account tides raised by the orbital motion of the companion, as well as interactions between tidal fields and NS spin. The main purpose of this work is to compute and study the Love numbers associated with this rotational-tidal response for slowly rotating NSs with realistic EoSs. Our main result is to show that existing universal relations between NS observables can be extended to include these Love numbers. These relations could be helpful for modelling and measuring spin corrections to tidal effects in the waveform, although the computation of such corrections is beyond the scope of this work.

In general relativity, the applied tidal field is described by two sets of symmetric-tracefree (STF) tensors [11, the gravitoelectric and gravitomagnetic multipole moments $\left\{\mathcal{E}_{L}\right\}_{\ell \geq 2}$ and $\left\{\mathcal{B}_{L}\right\}_{\ell \geq 2}$, where $L=a_{1} a_{2} \ldots a_{\ell}$ is a spatial

\footnotetext{
* jgagn129@uottawa.ca

$\dagger$ stephen.green@aei.mpg.de

$\ddagger$ landryp@uchicago.edu

$\S \overline{\text { nortiz@perimeterinstitute.ca }}$
} 
multi-index. These tidal moments are assumed to be sourced by an external mass and momentum distribution, such as a binary companion. At the linear level, neglecting the spin of the body, an $\ell$-pole gravitoelectric tidal field will induce as a tidal response a mass moment $k_{\ell}^{\text {el }} R^{2 \ell+1} \mathcal{E}_{L} / G$, while an $\ell$-pole gravitomagnetic tidal field will induce a current moment $k_{\ell}^{\mathrm{mag}} M R^{2 \ell} \mathcal{B}_{L} / c^{2}$ (modulo normalizations), where $M$ and $R$ are the mass and radius of the body. We refer to $k_{\ell}^{\mathrm{el}}$ and $k_{\ell}^{\mathrm{mag}}$ as the gravitoelectric and gravitomagnetic tidal Love numbers. The gravitoelectric tidal Love numbers reduce to the Love numbers of Newtonian theory in the weak-field limit, while the gravitomagnetic tidal Love numbers arise only in general relativity. Together, they provide a complete description of the deformation of a nonrotating body subject to a weak, slowly varying tidal field. The tidal Love numbers have been calculated for a variety of configurations, including polytropes [5, 8, 10, NSs with realistic EoSs [12 16] and quark stars [17, 18, and they have been shown to vanish identically for black holes $[8$. Several works have studied their impact on binary NS waveforms, showing a slight speed-up in the merger [12 16, 19, 24].

Neutron stars in binaries generically have nonzero spin $S^{a}$. This couples nonlinearly to the applied tidal field to generate additional corrections to the gravitational-wave phase. Observations indicate, however, that the dimensionless spin $\chi^{a} \equiv c S^{a} / G M^{2}$ is small- $\left|\chi^{a}\right| \lesssim 0.05[25]$ - so the effect is likely to be suppressed relative to the leading-order pure tidal deformation [26. Assuming low spin and rigid rotation, the leading-order rotational-tidal couplings are proportional to $\chi^{a} \mathcal{E}_{L}$ and $\chi^{a} \mathcal{B}_{L}$. These couplings combine the dipole spin vector with an $\ell$-pole tidal moment, generating bilinear moments of multipole orders $\ell-1 \leq \ell^{\prime} \leq \ell+1$. Just as a tidal Love number measures the amplitude of the gravitational field induced by a given tidal moment, a rotational-tidal Love number measures the field induced by a bilinear moment.

In this paper we restrict to quadrupolar applied tides. While spin couplings with the tidal quadrupole moments generate $\ell=1,2,3$ bilinear moments, the rotational-tidal response is in fact fully characterized by two octupole rotational-tidal Love numbers, $\mathfrak{f}^{\circ}$ (gravitoelectric) ${ }^{1}$ and $\mathfrak{k}^{\circ}$ (gravitomagnetic). Indeed, there are no $\ell=1$ Love numbers, as a dipole deformation represents an overall acceleration of the body, which can be eliminated by switching to its center-of-mass frame. Moreover, the $\ell=2$ Love numbers identified in Refs. [27 [29] can be eliminated by transforming to a suitably rotated frame, as we show in Appendix C Thus, the response of a rotating body to quadrupolar tides is characterized by the four Love numbers $\left\{k_{2}^{\mathrm{el}}, k_{2}^{\mathrm{mag}}, \mathfrak{f}^{\mathrm{o}}, \mathfrak{k}^{\mathrm{o}}\right\}$. These vanish for black holes [27], while for material bodies they depend on the EoS. Throughout this paper we will also use versions of the Love numbers scaled by powers of stellar compactness $G M / c^{2} R$. These will be denoted by uppercase letters $K_{2}^{\mathrm{el}} \equiv\left(c^{2} R / 2 G M\right)^{5} k_{2}^{\mathrm{el}}$, $\mathfrak{F}^{\circ} \equiv-\left(c^{2} R / 2 G M\right)^{5} \mathfrak{f}^{\circ}, K_{2}^{\text {mag }} \equiv\left(c^{2} R / 2 G M\right)^{4} k_{2}^{\text {mag }}$, and $\mathfrak{K}^{\circ} \equiv-\left(c^{2} R / 2 G M\right)^{5} \mathfrak{k}^{\circ}$. The scaled Love numbers are the quantities that enter into the universality relations [30], whereas the genuine Love numbers remain finite and nonzero in the zero-compactness limit, $G M / c^{2} R \rightarrow 0[27$.

Section II is devoted to justifying our restriction to quadrupolar applied tides. We show how the various tidal fields and Love numbers appear in the metric and we identify some of their physical effects. Supposing the tidal fields are sourced by a binary companion, we estimate the size of each term, and we argue that higher multipole terms make a smaller contribution to the metric. We note, however, that the higher- $\ell$ terms could still contribute significantly to the waveform itself, and this should be investigated in future work.

A complete analysis of the deformation of a slowly rotating body subject to a quadrupolar tidal field was carried out by Landry and Poisson [27 29, 31, and separately by Pani, Gualtieri, Maselli and Ferrari [26, 32, who also investigated the effect of an octupolar tidal field and worked to second order in spin. The two frameworks differ primarily in their assumptions about the fluid state: Pani, Gualtieri and Ferrari hold the fluid completely static 26, while Landry and Poisson allow it to develop tidal currents [10] in accordance with the circulation theorem of relativistic hydrodynamics 33. Because these fluid motions are vorticity-free in a nonrotating star, the latter state has been termed irrotational. The static state is incompatible with the Einstein equation except in axisymmetry [29]. In this work we follow the framework of Landry and Poisson, which we review in Sec. III.

In Sec. [V] we compute the four Love numbers for polytropes and an incompressible fluid, and we confirm that they agree with results of independent post-Newtonian calculations in the zero-compactness limit. We find that our results do not match those of Ref. [26], even in the regime where we expect fluid state differences to be insignificant. In particular, we observe no evidence of a sign change of $\mathfrak{F}^{\circ}$ (their $\delta \tilde{\lambda}_{M}^{32}$ ) at high compactness [26]. The $n=1$ polytrope results presented in Ref. [26] are also incompatible with our independent post-Newtonian calculation.

The octupole rotational-tidal Love numbers have been calculated for polytropes by Landry [28, and for realisticEoS NSs in the static fluid state by Pani, Gualtieri and Ferrari [26]. In Sec. [V], we perform the first-ever computation of $\mathfrak{f}^{\circ}$ and $\mathfrak{k}^{\circ}$ for realistic NSs in the irrotational state. The EoSs we adopt are piecewise polytropic fits to a subset of the candidate EoSs considered in Ref. 34]. Our models are chosen to be compatible with the maximum observed NS mass of approximately $2 M_{\odot}$ [35, 36], and they also respect the causal bound on the sound speed. We find that

\footnotetext{
${ }^{1}$ Note that we redefine this scaled Love number relative to Landry and Poisson 27 28]: fo [here] $\equiv \mathfrak{f}^{\mathrm{o}}[\mathrm{LP}]+\frac{5}{3} k_{2}^{\mathrm{el}}$ (see Sec. III F). Our new definition coincides with that of Ref. [26] up to an overall scale.
} 
these realistic EoSs have Love numbers that lie between those of the $n=0.5$ and $n=1$ polytropes, and we observe a qualitative difference between npe $\mu$ and exotic-matter EoSs.

One remarkable property of NSs is the existence of approximately EoS-independent relations between three macroscopic quantities: the moment of inertia $I$, the scaled Love number $K_{2}^{\mathrm{el}}$, and the rotational quadrupole moment $Q$. Although each quantity depends on the EoS in a separate way, Yagi and Yunes [30] discovered empirical functional relationships between them that are almost completely insensitive to the EoS. The origin of this I-Love- $Q$ universality is not entirely understood, although it has been linked to the emergence of isodensity contour self-similarity in compact stars [37. I-Love-Q relations have important applications in gravitational-wave astronomy. They imply that a precise measurement of one element of the triad is sufficient to determine the other two with percent-level accuracy. This can break the degeneracy between spin-spin interaction and rotational quadrupole contributions to the phasing of NS binary waveforms; an independent measurement of $K_{2}^{\mathrm{el}}$ could permit the extraction of the individual NS spins [38. Universal relations also have applications in parameter estimation and strong-field tests of general relativity [39]. Recent work has revealed that similar EoS-independent relations exist between the scaled gravitomagnetic and gravitoelectric tidal Love numbers [40, between the moment of inertia and the scaled gravitomagnetic tidal Love number $K_{2}^{\mathrm{mag}}$ 41, and between the scaled tidal Love numbers of two NSs in a binary system [42, 43, among other combinations (see Ref. 39] for a review).

In Sec. VI we extend I-Love universality to include the scaled rotational-tidal Love numbers. We show that these extended I-Love relations hold to within 2.5\% accuracy. This contrasts with Ref. [26], which found deviations from universality of up to $200 \%$. Extended I-Love universality also suggests the existence of universal relations among the scaled Love numbers. We confirm explicitly that these Love-Love relations hold between each pair of the four scaled Love numbers we study. Our findings provide further evidence that NSs can be characterized in an approximate way by a single number (beyond the mass and the spin) that determines their internal-structure dependent properties.

We also include several appendices. Appendix Adescribes our calculations of Love numbers in the post-Newtonian approximation. While general post-Newtonian formulas for the tidal Love numbers are known in the literature [10, only partial results exist for the rotational-tidal Love numbers; here, we derive expressions for $\mathfrak{f}^{\circ}$ and $\mathfrak{k}^{\circ}$ that are valid for any barotropic EoS. In Appendix B, we adapt the recipe presented in Sec. III to treat an incompressible fluid, which is a limiting case in terms of stiffness of the EoS. In Appendix C we show that the aforementioned quadrupole rotational-tidal Love numbers are spurious. And in Appendix D we derive the mapping between our Love numbers and those of Ref. [26].

Throughout this manuscript, lower case Latin indices $a, b, c, \ldots$ denote spatial components, and are raised and lowered with the Euclidean metric $\delta_{a b}$. Upper case Latin indices $A, B, C, \ldots$ denote angles $\theta^{A} \equiv(\theta, \phi)$, and are raised and lowered with the $\mathcal{S}^{2}$ metric $\mathcal{S}_{A B} \equiv \operatorname{diag}\left(1, \sin ^{2} \theta\right)$. Greek indices represent spacetime components. With the exception of Secs. I. II and Appendix A, we work in geometrized units $G=c=1$.

\section{TIDAL SCALES}

The rotational-tidal couplings we study in this paper are generated by the quadrupole moments $\mathcal{E}_{a b}, \mathcal{B}_{a b}$ of the tidal field. We work to first order in $\chi^{a}, \mathcal{E}_{a b}$ and $\mathcal{B}_{a b}$, and we also treat the second order bilinear terms $\chi^{a} \mathcal{E}_{b c}$ and $\chi^{a} \mathcal{B}_{b c}$. We claim that the bilinear octupole deformations associated with $\chi^{a} \mathcal{E}_{b c}$ and $\chi^{a} \mathcal{B}_{b c}$ represent important subleading corrections to the leading-order tides raised directly by the gravitoelectric quadrupole moment $\mathcal{E}_{a b}$. To justify this, in this section we describe the various scales of the problem, and we determine the relative sizes of the deformations induced by the tidal field.

We consider a body of mass $M$, radius $R$ and dimensionless spin angular momentum $\chi \equiv\left|\chi^{a}\right| \ll 1$ in a vacuum region of spacetime pervaded by the tidal influence of distant mass and momentum distributions. We imagine that these distributions are sourced by a binary companion of mass $M_{\mathrm{tid}}$ at a separation of $b \gg R$. (Alternately, $M_{\mathrm{tid}}$ and $b$ can be interpreted as generic mass and distance scales for the tidal source.) To leading order, the companion generates quadrupolar tidal fields $\mathcal{E}_{a b}, \mathcal{B}_{a b}$. These appear in the spacetime metric with the scalings [4]

$$
\frac{r^{2} \mathcal{E}_{a b}}{c^{2}} \sim \frac{G M_{\mathrm{tid}}}{c^{2} b}\left(\frac{r}{b}\right)^{2}, \quad \frac{r^{2} \mathcal{B}_{a b}}{c^{3}} \sim \frac{G M_{\mathrm{tid}}}{c^{2} b}\left(\frac{v}{c}\right)\left(\frac{r}{b}\right)^{2},
$$

where $r$ measures distance from the body's center of mass, $r \ll b$ in the neighborhood of the body and $v \sim \sqrt{G\left(M+M_{\text {tid }}\right) / b}$ is a velocity scale for the companion's orbital motion. We neglect the higher multipoles of the tidal field, such as the tidal octupole moments $\mathcal{E}_{a b c}, \mathcal{B}_{a b c}$, because they are suppressed by $(r / b)$ relative to the tidal quadrupole moments.

The couplings between $\chi^{a}$ and the tidal quadrupole moments produce terms of the form $\chi^{a} \mathcal{E}_{b c}, \chi^{a} \mathcal{B}_{b c}$ in the metric; they are suppressed by a factor of $\chi$ relative to those in Eq. (1). When $\chi \gg r / b$, these bilinear terms dominate over the octupole tidal terms. In this case, the further assumption that $r \sim G\left(M+M_{\mathrm{tid}}\right) / c^{2}$ in the neighborhood of the 
body implies that the dimensionless spin satisfies $v^{2} / c^{2} \ll \chi \ll 1$. This condition is naturally fulfilled when the binary separation is large and the component masses are broadly comparable. (If $\chi<v^{2} / c^{2}$, the bilinear terms are negligible and the tidal octupole terms are the important subleading corrections.)

By examining the form of the metric far from the tidally perturbed, slowly rotating body, we can determine the relative sizes of the deformations associated with each of the tidal and bilinear moments. The generic external metric ansatz was constructed by Landry and Poisson [27, 28. In an expansion in powers of $G M / c^{2} r$, the time-time and time-angle components of the metric are

$$
\begin{aligned}
g_{t t}= & -1+\frac{2 G M}{c^{2} r}-\left[1+\ldots+2 k_{2}^{\mathrm{el}}\left(\frac{R}{r}\right)^{5}(1+\ldots)\right] \frac{\mathcal{E}_{a b} x^{a} x^{b}}{c^{2}}+\frac{2 G M}{c^{2}}(1+\ldots) \frac{\chi^{b} \mathcal{B}_{a b} x^{a}}{c^{3}} \\
& -\frac{2 G M}{c^{2} r^{2}}\left[\frac{G M}{c^{2} r}+\ldots+2 \mathfrak{k}^{\mathrm{o}}\left(\frac{R}{r}\right)^{5}(1+\ldots)\right] \frac{\chi_{\langle a} \mathcal{B}_{b c\rangle} x^{a} x^{b} x^{c}}{c^{3}}, \\
g_{t A}= & \frac{2 G^{2} M^{2}}{c^{4} r^{3}} \epsilon_{a b c} x^{b} \chi^{c} x_{A}^{a}+\frac{2}{3}\left[1+\ldots-6\left(\frac{G M}{c^{2} r}\right) k_{2}^{\operatorname{mag}}\left(\frac{R}{r}\right)^{4}(1+\ldots)\right] \frac{\epsilon_{a c d} x^{c} \mathcal{B}^{d}{ }_{b} x^{b} x_{A}^{a}}{c^{3}} \\
& -\frac{2 G^{2} M^{2}}{c^{4} r}(1+\ldots) \frac{\epsilon_{a b c} x^{b} \mathcal{E}^{c}{ }_{d} \chi^{d} x_{A}^{a}}{c^{2}}-\frac{10 G M}{3 c^{2} r^{2}}\left[\frac{G^{2} M^{2}}{c^{4} r}+\ldots+\frac{6}{5} \mathfrak{f}^{\mathrm{o}}\left(\frac{R}{r}\right)^{5}(1+\ldots)\right] \frac{\epsilon_{a c}{ }^{d} x^{c} \mathcal{E}_{\langle d b} \chi_{e\rangle} x^{b} x^{e} x_{A}^{a}}{c^{2}} .
\end{aligned}
$$

in the Regge-Wheeler gauge and Boyer-Lindquist $(t, r, \theta, \phi)$ coordinates. Here, ellipses denote relativistic corrections of order $G M / c^{2} r$ and higher, $x^{a}$ are Cartesian mass-centred coordinates, $x_{A}^{a} \equiv \partial x^{a} / \partial \theta^{A}$ are their angular derivatives, and $\epsilon_{a b c}$ is the antisymmetric permutation symbol.

In $g_{t t}$ the set of terms proportional to $\mathcal{E}_{a b} x^{a} x^{b}$ describes a quadrupole deformation of the spacetime. The leading term in square brackets represents the applied gravitoelectric field, while the decaying term proportional to the Love number $k_{2}^{\text {el }}$ is the tidal response. This deformation is a Newtonian effect at leading order, as indicated by the factor of $c^{-2}$. The $t t$ component also contains a dipole deformation resulting from the coupling of the body's spin to the gravitomagnetic part of the tidal field; this is an overall acceleration of the body due to the Mathisson-Papapetrou spin force [45] 47, which enters as an order 1.5 post-Newtonian (1.5PN) correction by virtue of the factor of $c^{-5}$ [27]. The last set of terms in $g_{t t}$, proportional to $\chi_{\langle a} \mathcal{B}_{b c\rangle} x^{a} x^{b} x^{c}$, describes the octupole deformation of the spacetime due to another coupling of $\chi^{a}$ and $\mathcal{B}_{a b}$. The decaying piece of this solution - the tidal response -involves the rotationaltidal Love number $\mathfrak{k}^{\circ}$. This octupole deformation is a $1.5 \mathrm{PN}$ effect which is suppressed relative to the deformation associated with $k_{2}^{\mathrm{el}}$ by a factor of $\chi(v / c) G M / c^{2} r$.

Turning to the $t A$ component of the metric, the terms proportional to $\epsilon_{a c d} x^{c} \mathcal{B}^{d}{ }_{b} x^{b}$ represent a gravitomagnetic quadrupole deformation of the spacetime. The gravitomagnetic field is itself a 1PN phenomenon (as indicated by the $c^{-3}$ scaling in this time-space component), but the tidal response measured by $k_{2}^{\text {mag }}$ is suppressed by an additional factor of $G M / c^{2} r$ in the metric. The deformation associated with $k_{2}^{\mathrm{mag}}$ is therefore a $2 \mathrm{PN}$ effect, smaller than the gravitoelectric quadrupole deformation by a factor of $(v / c) G M / c^{2} r$. The set of terms proportional to $\epsilon_{a c}{ }^{d} x^{c} \mathcal{E}_{\langle d b} \chi_{e\rangle} x^{b} x^{e}$ in $g_{t A}$ corresponds to an octupole deformation generated by the coupling of the spin to the external gravitoelectric field; it enters at $1.5 \mathrm{PN}$ and is suppressed by $\chi G M / c^{2} r$ relative to the deformation associated with $k_{2}^{\text {el }}$. The amplitude of the decaying, tidal-response piece in square brackets is determined by $\mathfrak{f}^{\circ}$. The $t A$ component also contains a term proportional to $\epsilon_{a b c} x^{b} \chi^{c}$ describing the body's rotation, as well as a 2.5PN dipole deformation proportional to $\epsilon_{a b c} x^{b} \mathcal{E}_{d}^{c} \chi^{d}$ due to another spin force.

The structure of the metric allows us to remark on the expected scaling of the bilinear quadrupole deformations that would have been induced by couplings between $\chi^{a}$ and $\mathcal{E}_{a b c}, \mathcal{B}_{a b c}$, had we included the tidal octupole moments in our description. Because such bilinear terms are generated via octupole (rather than quadrupole) couplings, they will be suppressed relative to their counterparts in Eq. (2) by a factor of $r / b$, although they are of the same post-Newtonian order $(1.5 \mathrm{PN})$.

On the basis of these scalings, we conclude that the most important corrections to the leading-order tides measured by $k_{2}^{\text {el }}$ come from the deformations associated with $k_{2}^{\text {mag }}$ and $\mathfrak{f}^{\circ}$. They are smaller than the quadrupole induced by $\mathcal{E}_{a b}$ by factors of $(v / c)\left(G M / c^{2} r\right)$ and $\chi\left(G M / c^{2} r\right)$, respectively. Although $\chi \gg v^{2} / c^{2}$, the comparison of $\chi$ and $v / c$ is ambiguous, and depends on the parameters of the binary. Very early during binary inspiral,

$$
\frac{v}{c} \sim \frac{\left[G\left(M+M_{\mathrm{tid}}\right) f_{\mathrm{GW}}\right]^{1 / 3}}{c} \approx 0.06\left(\frac{M+M_{\mathrm{tid}}}{2 M_{\odot}}\right)^{1 / 3}\left(\frac{f_{\mathrm{GW}}}{25 \mathrm{~Hz}}\right)^{1 / 3},
$$

where $f_{\mathrm{GW}}$ is twice the orbital frequency; for a lightweight binary of total mass $\sim 2 M_{\odot}$, this is comparable to the maximum known spin for a NS in a binary that merges within the Hubble time, $\chi \approx 0.05$ [25]. Hence, the deformations produced by rotational-tidal couplings can be just as large as the tides due to gravitomagnetism. 
We remark that the preceding discussion does not mean that $k_{2}^{\text {mag }}$ and $f^{o}$ necessarily make the largest contributions (after $k_{2}^{\mathrm{el}}$ ) to the gravitational-wave tidal phase. Determining the precise corrections the rotational-tidal Love numbers make to the tidal phasing of the binary NS waveform is beyond the scope of this work.

\section{FRAMEWORK}

In this section we describe the approach of Landry and Poisson 27 29] for treating the deformation of a slowly and rigidly rotating NS caused by a weak, slowly varying quadrupolar tidal field. The idea is to solve the Einsteinfluid equations for a NS subject to asymptotic conditions corresponding to the applied field rather than the standard asymptotically flat conditions. One can then read off the induced field, whose amplitude determines the Love number, from the solution.

We consider a four-dimensional spacetime described by a metric tensor $g_{\alpha \beta}$, and we treat the NS matter as a perfect fluid with energy-momentum tensor

$$
T_{\alpha \beta}=(\mu+p) u_{\alpha} u_{\beta}+p g_{\alpha \beta}
$$

Here, $\mu$ and $p$ are the total fluid energy density and pressure, and $u^{\alpha}$ is the four-velocity of the fluid elements. The total energy density $\mu$ is the sum of the rest mass density $\rho$ and the internal thermodynamic energy $\epsilon$. We assume the fluid to be barotropic, with $\operatorname{EoS} p=p(\rho)$. The remaining fluid state variables follow from the EoS and the first law of thermodynamics for barotropic fluids,

$$
d(\epsilon / \rho)=-p d(1 / \rho)
$$

The matter and metric satisfy the Einstein equation and, for a one-parameter EoS, all of the hydrodynamic equations follow from energy-momentum conservation.

For weak tides and slow rotation, the spacetime and matter fields describing the NS and its neighborhood differ by a small amount from those of an isolated, nonrotating NS. This allows us to work in perturbation theory about a static, spherically symmetric background star. We describe the background solution in the following subsection.

In Sec. III B we write down the form of the tidally perturbed metric. This ansatz is constructed by adding terms to the background metric proportional to the moments of the applied tidal field and the spin of the star to interpolate between the star and the tidal environment, with radial dependence to be determined by the Einstein equation. We keep terms proportional to $\mathcal{E}_{a b}, \mathcal{B}_{a b}, \chi^{a}$, as well as the bilinear quantities $\chi^{a} \mathcal{E}_{b c}$ and $\chi^{a} \mathcal{B}_{b c}$.

To solve the Einstein equation it is convenient to split the problem into two parts, the interior and exterior regions of the star. We present the exterior solution (known analytically) in subsection III C. The exterior solution matches to the applied field far away, but also contains subleading parts with undetermined coefficients, the four scaled Love numbers $\left\{K_{2}^{\mathrm{el}}, K_{2}^{\mathrm{mag}}, \mathfrak{F}^{\mathrm{o}}, \mathfrak{K}^{\mathrm{o}}\right\}$. These quantities are determined by matching to the interior solution, which is required to be regular at the origin. We describe the matter part of the interior solution in Sec. IIID, and the solution for the metric - as well as the procedure for obtaining the Love numbers - in Sec. IIIE. The interior solution will typically be determined numerically, and it depends on the chosen fluid EoS.

We assume throughout that the dynamical time scale $\sqrt{b^{3} /\left(M+M_{\mathrm{tid}}\right)}$ of the tidal field is much longer than the characteristic time scale $\sqrt{R^{3} / M}$ of the internal stellar dynamics. This is a physically reasonable assumption for the inspiral stage of a binary NS system when the orbital separation is many times larger than the stellar radius. Accordingly, we treat the applied tides as stationary.

\section{A. Background solution}

The background solution is taken to be a static, spherically symmetric star with line element

$$
\overline{d s}^{2}=-e^{2 \psi(r)} d t^{2}+f(r)^{-1} d r^{2}+r^{2} d \mathcal{S}^{2}
$$

where $f(r) \equiv 1-2 m(r) / r$ and $d \mathcal{S}^{2} \equiv \mathcal{S}_{A B} d \theta^{A} d \theta^{B}$. The functions $\psi(r)$ and $m(r)$ are determined from the matter by the Einstein equation, which reduces to two ordinary differential equations (ODEs),

$$
\begin{aligned}
& \frac{d m}{d r}=4 \pi r^{2} \bar{\mu}, \\
& \frac{d \psi}{d r}=\frac{m+4 \pi r^{3} \bar{p}}{r^{2} f} .
\end{aligned}
$$


Here, and in the rest of Sec. III] we use overbars to denote background quantities. Conservation of energy-momentum, along with the above equations, yields the Tolman-Oppenheimer-Volkoff (TOV) equation,

$$
\frac{d \bar{p}}{d r}=-\frac{(\bar{\mu}+\bar{p})\left(m+4 \pi r^{3} \bar{p}\right)}{r^{2} f} .
$$

The equations of structure Eqs. (7)- $(9)$ are completed by the EoS. Outside the star, where $T_{\alpha \beta}=0$, the solution is Schwarzschild, with $f(r)=e^{2 \psi(r)}=1-2 M / r$. Inside the star $(0 \leq r \leq R)$, the equations of structure are solved subject to the regularity condition $m(0)=0$ at the center, and the matching conditions $\bar{p}(R)=0$ and $m(R)=M$ at the surface.

\section{B. Perturbed metric ansatz}

Following the detailed analysis of Refs. 27 29], the metric describing the tidally deformed, slowly rotating star is constructed by adding deformation terms to the unperturbed metric (6). To describe the pure tidal response, we add terms proportional to the applied quadrupolar tidal field $\mathcal{E}_{a b}$ and $\mathcal{B}_{a b}$. To describe the slow rotation we add a term proportional to the angular velocity $\Omega^{a} \equiv \chi^{a} M^{2} / I$, where $I$ is the moment of inertia. And to study the rotational-tidal response we add terms proportional to the bilinear quantities $\Omega^{a} \mathcal{E}_{b c}$ and $\Omega^{a} \mathcal{B}_{b c}$. These mixed terms are decomposed with respect to parity and multipole order (dipole, quadrupole, octupole) into the bilinear moments defined in Table [ All the terms are multiplied by functions of $r$ to be determined later using the field equations. The radial functions are designed to encapsulate the star's tidal response, and ensure that at large $r$ the metric asymptotes to that of the tidal environment [4].

The precise form of the perturbed metric is constructed such that it transforms suitably under parity and rotations. To do this, all moments - tidal, rotational, rotational-tidal - are repackaged into a set of potentials by taking duals and contracting with the unit radial vector $n^{a} \equiv x^{a} / r$ and its angular derivatives $n_{A}^{a} \equiv \partial n^{a} / \partial \theta^{A}$ (see Table II). These are inserted according to their transformation properties in the various components of the metric. In Regge-Wheeler gauge, the perturbed metric takes the form

$$
\begin{aligned}
g_{t t} & =-e^{2 \psi(r)}+e_{t t}^{\mathrm{q}}(r) \mathcal{E}^{\mathrm{q}}+k_{t t}^{\mathrm{d}}(r) \mathcal{K}^{\mathrm{d}}+k_{t t}^{\mathrm{o}}(r) \mathcal{K}^{\mathrm{o}}, \\
g_{t r} & =\hat{e}_{t r}^{\mathrm{q}}(r) \hat{\mathcal{E}}^{\mathrm{q}}+k_{t r}^{\mathrm{d}}(t, r) \mathcal{K}^{\mathrm{d}}+k_{t r}^{\mathrm{o}}(t, r) \mathcal{K}^{\mathrm{o}}, \\
g_{r r} & =f^{-1}+e_{r r}^{\mathrm{q}}(r) \mathcal{E}^{\mathrm{q}}+k_{r r}^{\mathrm{d}}(r) \mathcal{K}^{\mathrm{d}}+k_{r r}^{\mathrm{o}}(r) \mathcal{K}^{\mathrm{o}}, \\
g_{t A} & =[1-\omega(r)] r^{2} \Omega_{A}^{\mathrm{d}}+b_{t}^{\mathrm{q}}(r) \mathcal{B}_{A}^{\mathrm{q}}+\hat{b}_{t}^{\mathrm{q}}(t, r) \hat{\mathcal{B}}_{A}^{\mathrm{q}}+f_{t}^{\mathrm{d}}(r) \mathcal{F}_{A}^{\mathrm{d}}+f_{t}^{\mathrm{o}}(r) \mathcal{F}_{A}^{\mathrm{o}}, \\
g_{r A} & =\hat{b}_{r}^{\mathrm{q}}(r) \hat{\mathcal{B}}_{A}^{\mathrm{q}}, \\
g_{A B} & =r^{2} \mathcal{S}_{A B}+e^{\mathrm{q}}(r) \mathcal{S}_{A B} \mathcal{E}^{\mathrm{q}}+k^{\mathrm{o}}(r) \mathcal{S}_{A B} \mathcal{K}^{\mathrm{o}} .
\end{aligned}
$$

This form of the metric ensures that the perturbed Einstein equation will automatically decompose according to the potentials. Close examination of the metric shows that the star's spin appears only in $g_{t A}$, the pure gravitoelectric tide appears in the diagonal components, and the pure gravitomagnetic tide appears only in $g_{t A}$ [8].

Most of the coefficients of the potentials in Eq. (10) are functions of $r$ alone as a consequence of our assumption of stationary tides. However, some of them acquire a time dependence through gravitomagnetic induction inside the rotating star, even when $\mathcal{B}_{a b}$ is stationary 29 . Without the internal dynamics, the nonaxisymmetric part of the tidal response would violate the Einstein equation. Following Refs. 28, 29, we assume that the time dependence of the metric perturbations associated with $k_{t r}^{\mathrm{d}}, \hat{b}_{t}^{\mathrm{q}}$, and $k_{t r}^{\mathrm{o}}$ can be at most linear in $\Omega t$. The field equations then show that

$$
\begin{aligned}
k_{t r}^{\mathrm{d}}(t, r) & =t k_{t r 1}^{\mathrm{d}}(r), \\
\hat{b}_{t}^{\mathrm{q}}(t, r) & =t \hat{b}_{t 1}^{\mathrm{q}}(r), \\
k_{t r}^{\mathrm{o}}(t, r) & =t k_{t r 1}^{\mathrm{o}}(r) .
\end{aligned}
$$

While time dependence of some kind is physically required, the linear growth is an artifact of the perturbative expansion. Indeed, the time dependence has been shown to be bounded in a physical setting [28. The functions (11) vanish outside the star, so the exterior solution remains stationary.

\section{Exterior solution}

Outside the star, the energy-momentum tensor vanishes. Using the metric ansatz (10), and discarding terms of second or higher order in spin or tides, the vacuum Einstein equation decouples according to the potentials into ODEs. 


\begin{tabular}{cccc}
\hline \hline Moment & Definition & Parity & Multipole order $\ell$ \\
\hline $\mathcal{F}_{a}$ & $\mathcal{E}_{a b} \Omega^{b}$ & Odd & 1 \\
$\hat{\mathcal{E}}_{a b}$ & $2 \Omega^{c} \epsilon_{c d(a} \mathcal{E}^{d}{ }_{b)}$ & Even & 2 \\
$\mathcal{F}_{a b c}$ & $\mathcal{E}_{\langle a b} \Omega_{c\rangle}$ & Odd & 3 \\
$\mathcal{K}_{a}$ & $\mathcal{B}_{a b} \Omega^{b}$ & Even & 1 \\
$\hat{\mathcal{B}}_{a b}$ & $2 \Omega^{c} \epsilon_{c d(a} \mathcal{B}^{d}{ }_{b)}$ & Odd & 2 \\
$\mathcal{K}_{a b c}$ & $\mathcal{B}_{\langle a b} \Omega_{c\rangle}$ & Even & 3 \\
\hline \hline
\end{tabular}

TABLE I: Bilinear moments resulting from couplings of the dipole angular velocity vector $\Omega^{a}$ to the quadrupolar tidal field. Parentheses indicate symmetrization and angular brackets indicate the STF operation (symmetrization and removal of all traces).

\begin{tabular}{cc}
\hline \hline Potential & Definition \\
\hline$\Omega_{A}^{\mathrm{d}}$ & $\epsilon_{a b c} n^{b} \Omega^{c} n_{A}^{a}$ \\
$\mathcal{E}^{\mathrm{q}}$ & $\mathcal{E}_{a b} n^{a} n^{b}$ \\
$\mathcal{B}_{A}^{\mathrm{q}}$ & $\epsilon_{a b c} n^{b} \mathcal{B}^{c}{ }^{d} n^{d} n_{A}^{a}$ \\
$\mathcal{F}_{A}^{\mathrm{d}}$ & $\epsilon_{a b c} n^{b} \mathcal{F}^{c} n_{A}^{a}$ \\
$\hat{\mathcal{E}}^{\mathrm{q}}$ & $\hat{\mathcal{E}}_{a b} n^{a} n^{b}$ \\
$\hat{\mathcal{E}}_{A}^{\mathrm{q}}$ & $\left(\delta_{a}{ }^{b}-n_{a} n^{b}\right) \hat{\mathcal{E}}_{\text {bc }} n^{c} n_{A}^{a}$ \\
$\mathcal{F}_{A}^{\mathrm{o}}$ & $\epsilon_{a b c} n^{b} \mathcal{F}^{c}{ }_{d e} n^{d} n^{e} n_{A}^{a}$ \\
$\mathcal{\mathcal { B }}_{A}^{\mathrm{q}}$ & $\epsilon_{a b c} n^{b} \hat{\mathcal{B}}^{c}{ }^{a} n^{d} n_{A}^{a}$ \\
$\mathcal{K}^{\mathrm{d}}$ & $\mathcal{K}_{a} n^{a}$ \\
$\mathcal{K}_{A}^{\mathrm{d}}$ & $\left(\delta_{a}{ }^{b}-n_{a} n^{b}\right) \mathcal{K}_{b} n_{A}^{a}$ \\
$\mathcal{K}^{\mathrm{o}}$ & $\mathcal{K}_{a b c} n^{a} n^{b} n^{c}$ \\
$\mathcal{K}_{A}^{\mathrm{o}}$ & $\left(\delta_{a}{ }^{d}-n_{a} n^{d}\right) \mathcal{K}_{d b c} n^{b} n^{c} n_{A}^{a}$ \\
\hline \hline
\end{tabular}

TABLE II: Potentials appearing in the metric and fluid ansatzes of Eqs. 10 and $(15)$. The construction of the tidal potentials is described in Ref. [44] and the bilinear potentials in Refs. [27, 31].

These can then be integrated to obtain analytic expressions for the radial functions [27].

The function $\omega$ satisfies the ODE

$$
r \frac{d^{2} \omega}{d r^{2}}+4 \frac{d \omega}{d r}=0
$$

and we choose $\omega=1-2 I / r^{3}$ as the solution so that, in the absence of tides, the exterior reduces to the linearized Kerr spacetime. The solution contains a free parameter, the moment of inertia $I$, which must be determined by matching to the interior solution.

The equations for the radial functions associated with the tidal potentials $\mathcal{E}^{\mathrm{q}}$ and $\mathcal{B}_{A}^{\mathrm{q}}$ reduce to two secondorder homogeneous ODEs. One of them determines the function $e_{t t}^{\mathrm{q}}$, which is algebraically related to the other gravitoelectric-sector functions $\left\{e_{r r}^{\mathrm{q}}, e^{\mathrm{q}}\right\}$. The other governs the sole gravitomagnetic-sector function $b_{t}^{\mathrm{q}}$.

For each ODE, there exist two independent solutions: one decaying in powers of $r$, the other growing. The amplitude of the growing solution is set so that the spacetime outside the star matches onto the tidal environment at large $r$. The amplitude of the decaying solution - the tidal Love number - is set by matching to the regular interior solution at $r=R$. The exterior solutions for $e_{t t}^{\mathrm{q}}$ and $b_{t}^{\mathrm{q}}$, involving undetermined scaled Love numbers $K_{2}^{\mathrm{el}}$ and $K_{2}^{\mathrm{mag}}$, are listed in Table III

The radial functions associated with the bilinear potentials satisfy second-order inhomogeneous ODEs sourced by the functions $e_{t t}^{\mathrm{q}}, b_{t}^{\mathrm{q}}$ and $\omega$, which generate particular solutions in addition to the growing and decaying ones. Nevertheless, there are still two free parameters in each exterior solution, and they are set by the boundary conditions at the stellar surface and at large $r$. In this case, however, the coefficient of a decaying solution is not necessarily a Love number: some of the constants are pure gauge [27] (see Appendix C for an example). By carefully identifying and eliminating the gauge constants, one is left with two ODEs for the octupole radial functions: the first determines $f_{t}^{\circ}$, and the second determines $k_{t t}^{\mathrm{o}}$, which is algebraically related to $\left\{k_{r r}^{\mathrm{o}}, k^{\circ}\right\}$. Analytic expressions for these functionsinvolving the undetermined scaled rotational-tidal Love numbers $\mathfrak{F}^{\circ}$ and $\mathfrak{K}^{\circ}$-are given in Table III. The functions 


$$
\begin{aligned}
e_{t t}^{\mathrm{q}}= & -4 M^{2} x^{2}\left\{\left(1-\frac{1}{x}\right)^{2}+\frac{2}{x^{5}}\left[-30 x^{3}(x-1)^{2} \ln \left(1-\frac{1}{x}\right)-\frac{5}{2} x(2 x-1)\left(6 x^{2}-6 x-1\right)\right] K_{2}^{\mathrm{el}}\right\} \\
b_{t}^{\mathrm{q}}= & \frac{16 M^{3} x^{3}}{3}\left\{\left(1-\frac{1}{x}\right)-\frac{3}{x^{5}}\left[20 x^{4}(x-1) \ln \left(1-\frac{1}{x}\right)+\frac{5}{3} x\left(12 x^{3}-6 x^{2}-2 x-1\right)\right] K_{2}^{\mathrm{mag}}\right\} \\
k_{t t}^{\mathrm{o}}= & -4 I x^{2}\left\{\frac { 1 } { x ^ { 7 } } \left[-10 x^{4}(x-1)\left(280 x^{3}-420 x^{2}+140 x+3\right) \ln \left(1-\frac{1}{x}\right)-2800 x^{7}+5600 x^{6}-\frac{9100}{3} x^{5}+\frac{610}{3} x^{4}+\frac{115}{3} x^{3}+5 x^{2}-\frac{5}{6} x\right.\right. \\
& \left.\left.-\frac{5}{6}\right] K_{2}^{\mathrm{mag}}+\frac{2}{x^{6}}\left[-420 x^{4}(2 x-1)(x-1)^{2} \ln \left(1-\frac{1}{x}\right)-7 x^{2}\left(120 x^{4}-240 x^{3}+130 x^{2}-10 x-1\right)\right] \mathfrak{K}^{\mathrm{o}}+\frac{1}{2 x^{2}}-\frac{1}{2 x^{3}}\right\} \\
f_{t}^{\mathrm{o}}= & 8 I M x^{3}\left\{\frac{5}{4 x^{7}}\left[4 x^{3}\left(420 x^{5}-700 x^{4}+280 x^{3}+5 x-2\right) \ln \left(1-\frac{1}{x}\right)+\frac{2}{3} x^{2}\left(2520 x^{5}-2940 x^{4}+420 x^{3}+70 x^{2}+44 x+3\right)\right] K_{2}^{\mathrm{el}}\right. \\
& \left.+\frac{2}{x^{6}}\left[210 x^{5}(3 x-2)(x-1) \ln \left(1-\frac{1}{x}\right)+\frac{7}{2} x^{2}\left(180 x^{4}-210 x^{3}+30 x^{2}+5 x+1\right)\right] \mathfrak{F}^{\mathrm{o}}-\frac{5}{12 x^{3}}+\frac{1}{6 x^{4}}\right\}
\end{aligned}
$$

TABLE III: Select radial functions appearing in the $t t$ and $t A$ components of the exterior metric, expressed in terms of $x=r /(2 M)$. All functions within square brackets behave as $1+\mathcal{O}(1 / x)$ when $x \gg 1$. We remark that our expression for $f_{t}^{\circ}$ differs from that of Refs. [27, 28] because of our redefinition of $\mathfrak{F}^{\circ}$ (see Sec. IIIF).

$\left\{k_{t r}^{\mathrm{d}}, \hat{b}_{t}^{\mathrm{q}}, k_{t r}^{\mathrm{o}}\right\}$ vanish by virtue of the vacuum Einstein equation.

The result of this discussion is that only a subset $\left\{e_{t t}^{\mathrm{q}}, b_{t}^{\mathrm{q}}, k_{t t}^{\mathrm{o}}, f_{t}^{\mathrm{o}}\right\}$ of the external radial functions are needed to compute the Love numbers. These functions appear solely in the $t t$ and $t A$ components of the metric. For the complete exterior solution to the problem, we refer the reader to Ref. [27.

\section{Perturbed fluid}

The interior solution is governed by the hydrodynamic equations. In this subsection, we use these equations and the Einstein equation to cast the fluid variables in terms of the radial functions from the metric ansatz.

We begin by decomposing the perturbed fluid variables $\mu, p, u_{r}$ and $u_{A}$ in terms of the tidal and bilinear potentials of Table II] as was done for the metric ansatz. (The time component of the fluid four-velocity is automatically fixed by properly normalizing $u^{\alpha}$.) The decomposition is presented in detail in Refs. [28, 29].

We next impose energy-momentum conservation. For an unperturbed barotrope satisfying the first law of thermodynamics (5), this reduces to the conservation statement $\mathcal{L}_{u} \omega_{\alpha \beta}=0$, where $\mathcal{L}_{u}$ is a Lie derivative along $u^{\alpha}$. In other words, the vorticity tensor $\omega_{\alpha \beta} \equiv \partial_{\alpha}\left(h u_{\beta}\right)-\partial_{\beta}\left(h u_{\alpha}\right)$ is conserved along the fluid wordlines. Here, $h \equiv(\mu+p) / \rho$ is the specific enthalpy. Taking a variation, and assuming that the EoS is unchanged by the perturbation, it follows that

$$
\mathcal{L}_{u} \Delta \omega_{\alpha \beta}=0
$$

where $\Delta \omega_{\alpha \beta}$ denotes the Lagrangian perturbation of the vorticity tensor (see Sec. 7.4.2 of Ref. [48] for a derivation).

We suppose that the tidally deformed, slowly rotating star began in an unperturbed state at $t=0$, and that the tidal field was switched on adiabatically. Equation 13 then implies that

$$
\Delta \omega_{\alpha \beta}=0
$$

for all time. This is the natural state that arises in a binary system that was widely separated in the distant past. The vorticity preservation condition (14) almost completely constrains the perturbed fluid variables. Apart from the freedom to add stationary $r$ - and $g$-modes, which we suppress following Refs. [28, 29], there remains some freedom in $u_{A}$. This freedom is fixed by the Einstein equation, and all the perturbed fluid variables can be related to the radial functions appearing in Eq. 10.

We impose Eq. (14) to place the NS in a vorticity-preserving state, which we call the irrotational state because its vorticity vanishes in the $\Omega \rightarrow 0$ limit. After a lengthy calculation [28, 29], the full expressions for the perturbed fluid 
variables are found to be

$$
\begin{aligned}
\mu= & \bar{\mu}+\frac{1}{2} e^{-2 \psi}(\bar{\mu}+\bar{p}) \frac{d \bar{\mu}}{d \bar{p}} e_{t t}^{\mathrm{q}} \mathcal{E}^{\mathrm{q}}, \\
p= & \bar{p}+\frac{1}{2} e^{-2 \psi}(\bar{\mu}+\bar{p}) e_{t t}^{\mathrm{q}} \mathcal{E}^{\mathrm{q}} \\
u_{r}= & e^{-\psi} \hat{e}_{t r}^{\mathrm{q}} \hat{\mathcal{E}}^{\mathrm{q}}-t e^{-\psi}\left[\frac{1}{8 \pi r^{2}(\bar{\mu}+\bar{p})}-1\right] k_{t r 1}^{\mathrm{d}} \mathcal{K}^{\mathrm{d}}-t e^{-\psi}\left[\frac{3}{4 \pi r^{2}(\bar{\mu}+\bar{p})}-1\right] k_{t r 1}^{\mathrm{o}} \mathcal{K}^{\mathrm{o}}, \\
u_{A}= & -e^{-\psi} r^{2} \omega \Omega_{A}^{\mathrm{d}}-\frac{1}{6} r^{2} e^{-3 \psi}\left(1-\omega+\frac{d \bar{\mu}}{d \bar{p}}\right) e_{t t}^{\mathrm{q}} \hat{\mathcal{E}}_{A}^{\mathrm{q}}+e^{-\psi} f_{t}^{\mathrm{d}} \mathcal{F}_{A}^{\mathrm{d}}+e^{-\psi} f_{t}^{\mathrm{o}} \mathcal{F}_{A}^{\mathrm{o}} \\
& +\frac{1}{3} t e^{-\psi} \omega b_{t}^{\mathrm{q}} \hat{\mathcal{B}}_{A}^{\mathrm{q}}-\frac{t e^{-\psi}}{16 \pi r^{2}(\bar{\mu}+\bar{p})}\left\{r^{2} f \frac{d k_{t r 1}^{\mathrm{d}}}{d r}+2\left[m-2 \pi r^{3}(\bar{\mu}-\bar{p})\right] k_{t r 1}^{\mathrm{d}}\right\} \mathcal{K}_{A}^{\mathrm{d}} \\
& -\frac{3 t e^{-\psi}}{16 \pi r^{2}(\bar{\mu}+\bar{p})}\left\{r^{2} f \frac{d k_{t r 1}^{\mathrm{o}}}{d r}+2\left[m-2 \pi r^{3}(\bar{\mu}-\bar{p})\right] k_{t r 1}^{\mathrm{o}}\right\} \mathcal{K}_{A}^{\mathrm{o}} .
\end{aligned}
$$

The time dependent terms in the fluid velocity represent dynamical currents induced by the stationary gravitomagnetic tidal field; they are tied to the time dependent radial functions introduced in Sec. IIIB. The linear dependence on $t$ may be viewed as a consequence of our slow rotation approximation.

\section{E. Interior solution}

With the ansatzes $(10)$ and $(15)$ for the metric and fluid variables, the perturbed Einstein equation is solved in the stellar interior. The undetermined radial functions in the metric satisfy generically inhomogeneous second-order ODEs. Thus, there exist two independent homogeneous solutions, plus a particular solution, for each differential equation. We demand that the solution be regular at the origin, and that the interior solution match the exterior one at the surface, up to first derivatives. While the system may appear to be overdetermined at first glance, with three conditions on two free parameters, we recall that the exterior solution also has a free parameter: the Love number (or the moment of inertia in the case of $\omega$ ). The matching condition fixes this final parameter, and determines the tidal response.

In general, the interior ODEs must be integrated numerically, as they depend on the fluid EoS, and we implement a shooting method to obtain the solutions. We perform a local analysis of each ODE near $r=0$ to determine the regularity conditions, and we then integrate outwards to the surface. Here, the matching conditions determine the amplitude of the regular solution and the free parameter of the exterior solution.

Consider, for example, the rotation. The function $\omega(r)$ satisfies

$$
r f \frac{d^{2} \omega}{d r^{2}}+\left[4 f-4 \pi r^{2}(\bar{\mu}+\bar{p})\right] \frac{d \omega}{d r}-16 \pi r(\bar{\mu}+\bar{p}) \omega=0
$$

inside the star. At the surface, $\omega$ matches on to the external solution $\omega(r>R)=1-2 I / r^{3}$. Writing ${ }^{\prime} \equiv d / d r$, regularity of $\omega$ at the origin requires $\omega^{\prime}(0)=0$, with $\omega(0)$ set by matching to the external solution. The matching conditions also determine the specific value of $I$ that appears in the exterior metric.

In the remainder of this section, we detail our method for calculating the four scaled Love numbers $\left\{K_{2}^{\mathrm{el}}, K_{2}^{\mathrm{mag}}, \mathfrak{F}^{\circ}, \mathfrak{K}^{\circ}\right\}$. Like in the external problem, the relevant ODEs involve only a small number of radial functions. In addition to the set $\left\{e_{t t}^{\mathrm{q}}, b_{t}^{\mathrm{q}}, k_{t t}^{\mathrm{o}}, f_{t}^{\mathrm{o}}\right\}$ from above, we also require $k_{t r 1}^{\mathrm{o}}$, which is non-vanishing in the interior and appears with $k_{t t}^{\mathrm{o}}$ in a coupled system of differential equations.

\section{Gravitoelectric sector: $K_{2}^{\text {el }}$ and $\mathfrak{F}^{o}$}

The scaled gravitoelectric tidal Love number $K_{2}^{\mathrm{el}}$ is determined by solving for the radial function $e_{t t}^{\mathrm{q}}$, which appears in $g_{t t}$. In the interior of the star, this function satisfies the ODE [28]

$$
r^{2} f \frac{d^{2} e_{t t}^{\mathrm{q}}}{d r^{2}}-2\left[\frac{3 m}{r}-1+2 \pi r^{2}(\bar{\mu}+3 \bar{p})\right] r \frac{d e_{t t}^{\mathrm{q}}}{d r}-2\left[3-2 \pi r^{2}(\bar{\mu}+\bar{p})\left(3+\frac{d \bar{\mu}}{d \bar{p}}\right)\right] e_{t t}^{\mathrm{q}}=0 .
$$

We would like to find a regular solution to this equation that matches the corresponding external expression given in Table III up to first derivatives at $r=R$. Local analysis of Eq. (17) near $r=0$ shows that the regular solution has 
$e_{t t}^{\mathrm{q}}(0)=e_{t t}^{\mathrm{q} \prime}(0)=0$, so its amplitude is determined by $e_{t t}^{\mathrm{q} \prime \prime}(0)$. The matching conditions at the surface then yield the value of $K_{2}^{\mathrm{el}}$.

The scaled gravitoelectric rotational-tidal Love number $\mathfrak{F}^{\circ}$ is calculated from the solution for $f_{t}^{\circ}$, which satisfies 28$]$

$$
\begin{aligned}
0= & r^{2} f \frac{d^{2} f_{t}^{\mathrm{o}}}{d r^{2}}-4 \pi r^{3}(\bar{\mu}+\bar{p}) \frac{d f_{t}^{\mathrm{o}}}{d r}+4\left[\frac{m}{r}-3+2 \pi r^{2}(\bar{\mu}+\bar{p})\right] f_{t}^{\mathrm{o}} \\
& -16 \pi r^{2}(\bar{\mu}+\bar{p}) f_{t}^{\mathrm{o}}+r^{3} e^{-2 \psi}(1-\omega)\left(\frac{9 m}{r}-2+20 \pi r^{2} \bar{p}\right) \frac{d e_{t t}^{\mathrm{q}}}{d r} \\
& +2 r^{2} e^{-2 \psi}\left[\left(\frac{5 m}{r}+2\right)(1-\omega)+2 \pi r^{2}(\bar{\mu}+\bar{p})\left(6+\frac{d \bar{\mu}}{d \bar{p}}\right) \omega+2 \pi r^{2}(\bar{\mu}+\bar{p})\left(\frac{d \bar{\mu}}{d \bar{p}}-2\right)\right] e_{t t}^{\mathrm{q}}
\end{aligned}
$$

in the interior. At the center, Eq. (18) yields a regular solution $f_{t}^{\mathrm{o}} \propto r^{4}$. The matching conditions at the surface then determine $\mathfrak{F}^{\circ}$

\section{Gravitomagnetic sector: $K_{2}^{\text {mag }}$ and $\mathfrak{K}^{o}$}

The scaled Love numbers $K_{2}^{\mathrm{mag}}$ and $\mathfrak{K}^{\mathrm{o}}$ are calculated in a similar fashion as $K_{2}^{\mathrm{el}}$ and $\mathfrak{F}^{\mathrm{o}}$. The scaled gravitomagnetic tidal Love number is determined by solving for the radial function $b_{t}^{\mathrm{q}}$, which satisfies [29]

$$
r^{2} f \frac{d^{2} b_{t}^{\mathrm{q}}}{d r^{2}}-4 \pi r^{3}(\bar{\mu}+\bar{p}) \frac{d b_{t}^{\mathrm{q}}}{d r}-2\left[3-\frac{2 m}{r}-4 \pi r^{2}(\bar{\mu}+\bar{p})\right] b_{t}^{\mathrm{q}}=0
$$

The regularity condition derived from a local analysis of $(19)$ is $b_{t}^{\mathrm{q}} \propto r^{3}$ near $r=0$. The matching procedure at $r=R$ yields the value of $K_{2}^{\text {mag }}$.

The calculation of $\mathfrak{K}^{\circ}$ is slightly more complicated, since two coupled ODEs must be solved. First, we determine the radial function $k_{t r 1}^{\mathrm{o}}$ throughout the star. It satisfies the ODE [29]

$$
\begin{aligned}
0= & r^{2} f \frac{d^{2} k_{t r 1}^{\mathrm{o}}}{d r^{2}}+\left[3\left(m-4 \pi r^{3} \bar{\mu}\right)+\left(m+4 \pi r^{3} \bar{p}\right) \frac{d \bar{\mu}}{d \bar{p}}\right] \frac{d k_{t r 1}^{\mathrm{o}}}{d r} \\
& -\frac{2}{r^{2} f}\left\{2\left[3-5 \pi r^{2}(\bar{\mu}+\bar{p})+8 \pi^{2} r^{4} \bar{p}^{2}\right] r^{2}-2\left[5-2 \pi r^{2}(5 \bar{\mu}+7 \bar{p})\right] r m-3 m^{2}-\left(m+4 \pi r^{3} \bar{p}\right)^{2} \frac{d \bar{\mu}}{d \bar{p}}\right\} k_{t r 1}^{\mathrm{o}} \\
& -\frac{32 \pi}{3} r^{2}(\bar{\mu}+\bar{p}) \omega \frac{d b_{t}^{\mathrm{q}}}{d r}+\frac{16 \pi}{3}(\bar{\mu}+\bar{p})\left[r^{2} \frac{d \omega}{d r}+2 \frac{3 r-7 m-4 \pi r^{3} \bar{p}}{f} \omega\right] b_{t}^{\mathrm{q}} .
\end{aligned}
$$

Since $k_{t r 1}^{\mathrm{o}}$ vanishes outside the star, the matching conditions at the surface are simply $k_{t r 1}^{\mathrm{o}}(R)=k_{t r 1}^{\mathrm{o}}{ }^{\prime}(R)=0$. $(\mathrm{Note}$ that these boundary conditions do not overdetermine the system [29.) Based on a local analysis of Eq. 20, the regular solution has $k_{t r 1}^{\mathrm{o}} \propto r^{4}$ at the origin.

Second, we solve for the radial function $k_{t t}^{o}$, which satisfies [29]

$$
\begin{aligned}
0= & r^{2} f \frac{d^{2} k_{t t}^{\mathrm{o}}}{d r^{2}}+2\left[1-\frac{3 m}{r}-2 \pi r^{2}(\bar{\mu}+3 \bar{p})\right] r \frac{d k_{t t}^{\mathrm{o}}}{d r}+4\left[\pi r^{2}(\bar{\mu}+\bar{p})\left(3+\frac{d \bar{\mu}}{d \bar{p}}\right)-3\right] k_{t t}^{\mathrm{o}}+\frac{1}{2} r^{2} f\left(\frac{d \bar{\mu}}{d \bar{p}}-1\right) \frac{d k_{t r 1}^{\mathrm{o}}}{d r} \\
& +\left\{\left(11+\frac{d \bar{\mu}}{d \bar{p}}\right) m+2 \pi r^{3}\left[(\bar{\mu}+7 \bar{p})-(\bar{\mu}-\bar{p}) \frac{d \bar{\mu}}{d \bar{p}}\right]-4 r\right\} k_{t r 1}^{\mathrm{o}}+S_{1} r \frac{d b_{t}^{\mathrm{q}}}{d r}+S_{0} b_{t}^{\mathrm{q}}
\end{aligned}
$$

\footnotetext{
${ }^{2}$ As mentioned in Ref. [28, the value of $\mathfrak{F}^{\circ}$ is sensitive to the presence of stellar $r$-modes; such modes would modify Eq. 18. The values of $\mathfrak{F}^{\circ}$ computed here correspond to stars free of $r$-modes.
} 


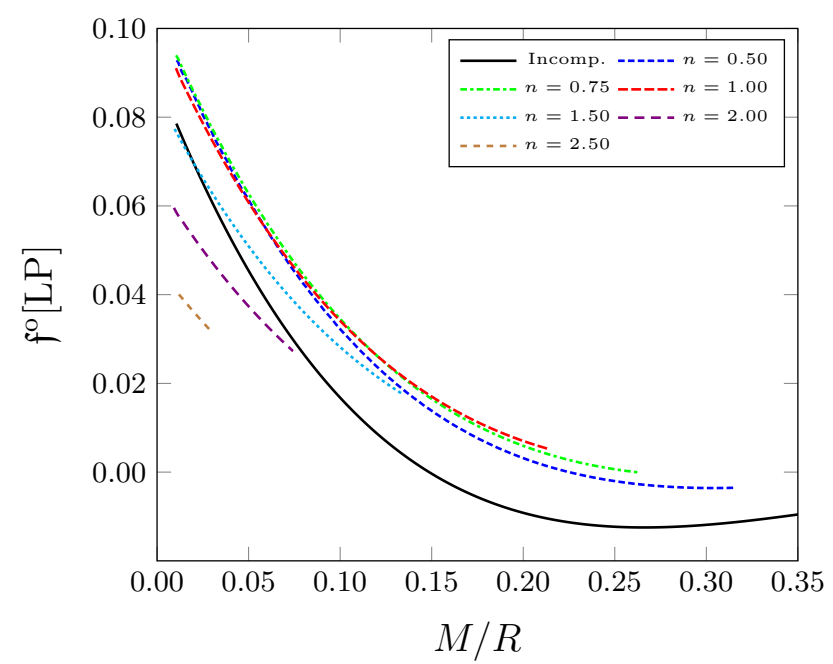

FIG. 1: Gravitoelectric rotational-tidal Love number $\mathfrak{f}^{\circ}[\mathrm{LP}]$ as a function of compactness. This is a reproduction of Fig. 1 of Ref. 28, with the addition of the incompressible fluid results. We employ a different definition of $\mathfrak{f}^{\circ}$ elsewhere in the paper - see Eq. (23).

with

$$
\begin{aligned}
S_{1}= & -\frac{2}{3}\left\{\frac{5 m^{2}}{r^{2}}+3-16 \pi^{2} r^{4} \bar{p}^{2}+4 \pi r^{2} \bar{\mu}-\frac{m}{r}\left[9+8 \pi r^{2}(\bar{\mu}+\bar{p})\right]\right\} r \frac{d \omega}{d r} \\
& -\frac{4}{3}\left\{3-\frac{m}{r}\left[9-4 \pi r^{2}(\bar{\mu}+\bar{p})\right]-4 \pi r^{2} \bar{p}\left[3-4 \pi r^{2}(\bar{\mu}+\bar{p})\right]\right\} \omega+4\left(1-\frac{3 m}{r}-4 \pi r^{2} \bar{p}\right), \\
S_{0}= & \frac{2}{3}\left\{\frac{10 m^{2}}{r^{2}}+4 \pi r^{2}\left[\left(3-8 \pi r^{2} \bar{p}\right) \bar{p}+2 \bar{\mu}\right]-\frac{m}{r}\left[3+16 \pi r^{2}(\bar{\mu}+\bar{p})\right]\right\} r \frac{d \omega}{d r} \\
& +\frac{4}{3}\left\{\frac{m}{r}\left[6+8 \pi r^{2}(\bar{\mu}+\bar{p})\right]+\left[9-4 \pi r^{2}(\bar{\mu}+\bar{p})\left(6+\frac{d \bar{\mu}}{d \bar{p}}-8 \pi r^{2} \bar{p}\right)\right]\right\} \omega \\
& -4\left\{\frac{2 m}{r}-\left[2 \pi r^{2}(\bar{\mu}+\bar{p})\left(1+\frac{d \bar{\mu}}{d \bar{p}}\right)-3\right]\right\} .
\end{aligned}
$$

A local analysis of Eq. (21) reveals that the regular solution has $k_{t t}^{o} \propto r^{3}$ near the origin. Finally, the matching conditions at the surface determine $\mathfrak{K}^{\circ}$.

\section{F. Redefinition of $\mathfrak{F}^{\circ}$}

As noted in the introduction, the definition of the scaled gravitoelectric rotational-tidal Love number $\mathfrak{F}^{\circ}$ given in this work differs from that of Landry and Poisson [27, 28; namely,

$$
\mathfrak{F}^{\mathrm{o}}[\text { here }]=\mathfrak{F}^{\mathrm{o}}[\mathrm{LP}]-\frac{5}{3} K_{2}^{\mathrm{el}}, \quad \text { which implies } \quad \mathfrak{f}^{\mathrm{o}}[\text { here }]=\mathfrak{f}^{\mathrm{o}}[\mathrm{LP}]+\frac{5}{3} k_{2}^{\mathrm{el}} .
$$

We claim that our new definition is more consistent with the interpretation of $\mathfrak{F}^{\circ}$ as a scaled rotational-tidal Love number.

With Landry and Poisson's definition of $\mathfrak{F}^{\circ}$, the octupole part of the $t A$ component of the exterior metric takes the schematic form

$$
g_{t A}^{\ell=3}=-\frac{10 M}{3 r^{2}}\left[\frac{M^{2}}{r}+\ldots+\frac{6}{5} \mathfrak{f}^{\mathrm{o}}[\mathrm{LP}]\left(\frac{R}{r}\right)^{5}(1+\ldots)+2 k_{2}^{\mathrm{el}}\left(\frac{R}{r}\right)^{5}(1+\ldots)\right] \epsilon_{a c}{ }^{d} x^{c} \mathcal{E}_{\langle d b} \chi_{c\rangle} x^{b} x^{e} x_{A}^{a},
$$

rather than that of Eq. (2b). (This expression can be obtained by expanding the radial function $f_{t}^{\circ}$ from Table IV of Ref. [27] in powers of $M / r$.) We see that there are two separate pieces that decay in $r$ at the same rate, which means 


\begin{tabular}{ccccc}
\hline \hline EoS & $k_{2}^{\mathrm{el}}$ & $k_{2}^{\text {mag }}\left(\times 10^{-3}\right)$ & $\mathfrak{f}^{\mathrm{o}}$ & $\mathfrak{k}^{\mathrm{o}}$ \\
\hline Incompressible & 0.7500 & -14.29 & 1.339 & 0.2083 \\
$\mathrm{n}=0.50$ & 0.4492 & -10.04 & 0.8539 & 0.1385 \\
$\mathrm{n}=0.75$ & 0.3434 & -8.330 & 0.6770 & 0.1124 \\
$\mathrm{n}=1.00$ & 0.2599 & -6.850 & 0.5334 & 0.09050 \\
$\mathrm{n}=1.50$ & 0.1433 & -4.478 & 0.3235 & 0.05740 \\
$\mathrm{n}=2.00$ & 0.07394 & -2.763 & 0.1886 & 0.03501 \\
$\mathrm{n}=2.50$ & 0.03485 & -1.575 & 0.1044 & 0.02026 \\
\hline \hline
\end{tabular}

TABLE IV: Post-Newtonian values of the Love numbers for the EoSs considered in Fig. 2, calculated with the methods of Appendix A.

that $\mathfrak{F}^{\circ}[\mathrm{LP}]$ is only partially measuring the response of the body to the spin-coupled gravitoelectric field. The fact that the $\mathfrak{F}^{\mathrm{o}}[\mathrm{LP}]$ and $K_{2}^{\mathrm{el}}$ terms in Eq. (24) have the same scaling with $r$, however, allows us to shift the rotational-tidal Love number as in Eq. (23) so that it fully captures the response.

The unnaturalness of Landry and Poisson's definition is clearly demonstrated in Fig. 1, reproduced from Ref. 28] with the addition of the incompressible fluid results. We see that the polytrope fo[LP] vs. compactness curves intersect one another and do not tend monotonically to the incompressible fluid. This is contrary to physical intuition, and it differs qualitatively from results for the other three Love numbers (see Fig. 2). These discrepancies disappear when our new definition for the rotational-tidal Love number is used, as Fig. 2c shows.

We have two additional comments on our definition of $\mathfrak{F}^{\circ}$. First, the shift in Eq. (23) has the same effect in the light-cone gauge [49] employed in Ref. 27] as it does here. This confirms that our new definition is not just a quirk of the Regge-Wheeler gauge. Second, our new definition of $\mathfrak{F}^{\circ}$ coincides with the scaled rotational-tidal Love number $\delta \tilde{\lambda}_{M}^{(32)}$ of Ref. [26] up to a constant factor [28].

\section{LOVE NUMBERS FOR POLYTROPES}

In this section, we test our methods by computing Love numbers for polytropes. We show that our results are consistent with similar calculations in the literature, and that when we take suitable limits they match post-Newtonian and incompressible fluid calculations. The consistency checks we perform are important as they provide support for our findings, which disagree with those of Ref. [26].

In Fig. 2 we plot the Love numbers for various polytropes as a function of stellar compactness. We use the mass polytrope $\operatorname{EoS} p(\rho)=K \rho^{1+1 / n}$, where $n>0$ is the polytropic index and $K$ is a constant $?^{3}$ A choice of $n$ and central pressure-to-density ratio $p_{c} / \rho_{c}=K \rho_{c}^{1 / n}$ uniquely specifies the stellar model. The Love numbers are plotted up to the maximum value of $M / R$ for which the given polytrope is stable against radial perturbations, as per the turning point criterion (see e.g., Ref. 48]). Our tidal Love number results agree with Refs. [8] and [10, while our rotational-tidal Love numbers match those of Ref. [28] (modulo the redefinition of $\mathfrak{F}^{\circ}$ ).

Figure 2 also includes Love numbers for incompressible fluid stars. The incompressible fluid constitutes the (singular) $n \rightarrow 0$ limit of the polytropic EoS. We see that the polytrope curves tend monotonically toward that of the incompressible fluid as $n$ decreases. The fact that the incompressible fluid possesses the largest Love numbers (in magnitude) of all the models is consistent with the physical intuition that, as the stiffest possible EoS, it should have the weakest internal gravity and hence the greatest deformability. Subtleties associated with the calculation of Love numbers for this uniform-density model are treated in Appendix B.

We also show polytrope and incompressible fluid Love numbers computed in post-Newtonian theory (indicated by circles on the vertical axes). Details of these independent calculations are provided in Appendix A and the results are listed in Table IV. It is apparent from Fig. 2 that the general-relativistic Love numbers match the post-Newtonian values in the weak-field limit $M / R \rightarrow 0$, as expected. This agreement further validates our Love number computations.

We now make a direct comparison of our results for the rotational-tidal Love numbers with those of Ref. [26. Pani, Gualtieri and Ferrari employ slightly different definitions for the Love numbers, but we derive the relation between the two conventions in Appendix D. The scaled gravitoelectric and gravitomagnetic rotational-tidal Love numbers of Ref. [26], respectively $\delta \tilde{\lambda}_{M}^{(32)}$ and $\delta \tilde{\lambda}_{E}^{(32)}$, are merely rescaled by a constant factor relative to our own definitions:

\footnotetext{
${ }^{3}$ Here, $p$ and $\rho$ refer to the background pressure and rest mass density. From this section on, we deal only with unperturbed fluid quantities, and we therefore drop the overbars on the background $\mu$ and $p$.
} 


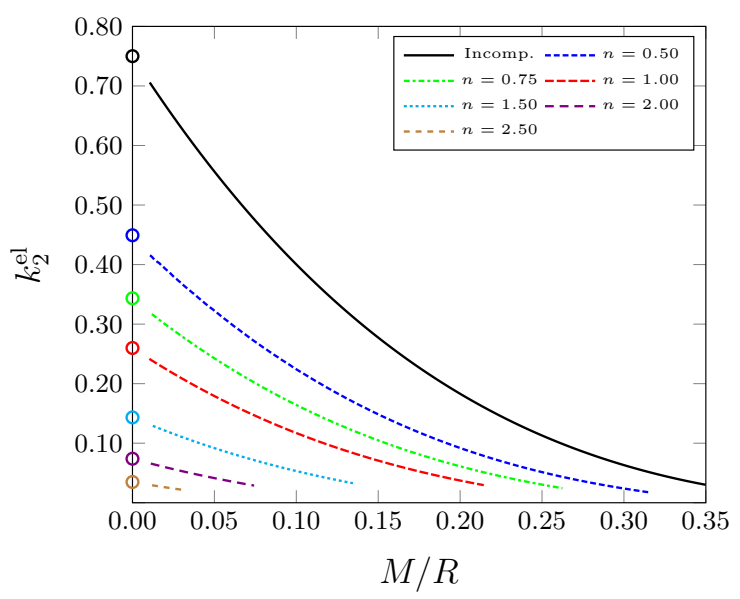

(a) Gravitoelectric tidal Love number

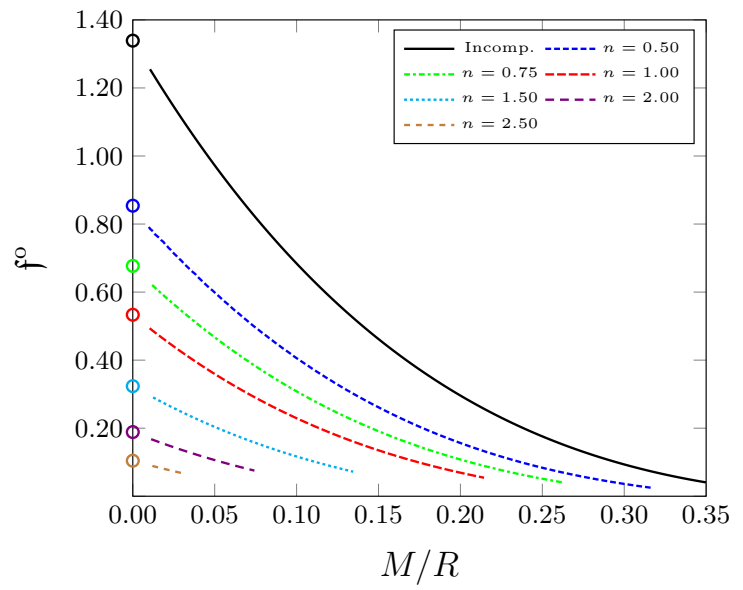

(c) Gravitoelectric rotational-tidal Love number

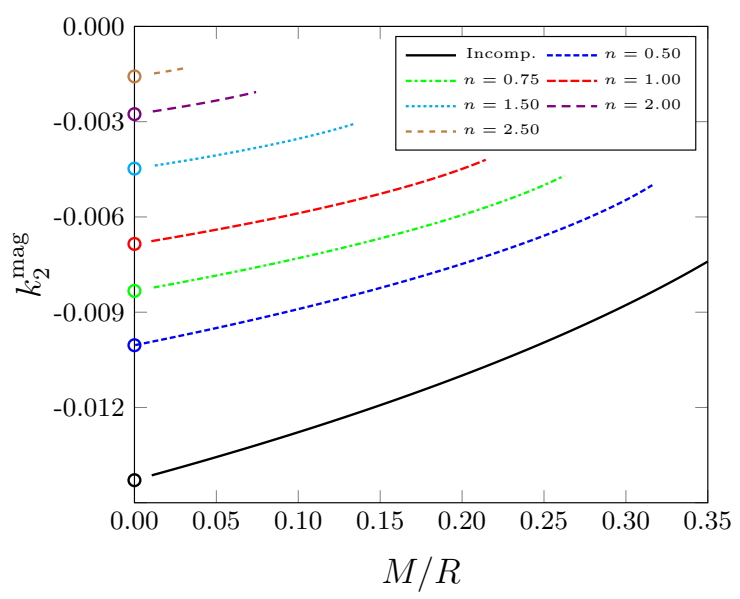

(b) Gravitomagnetic tidal Love number

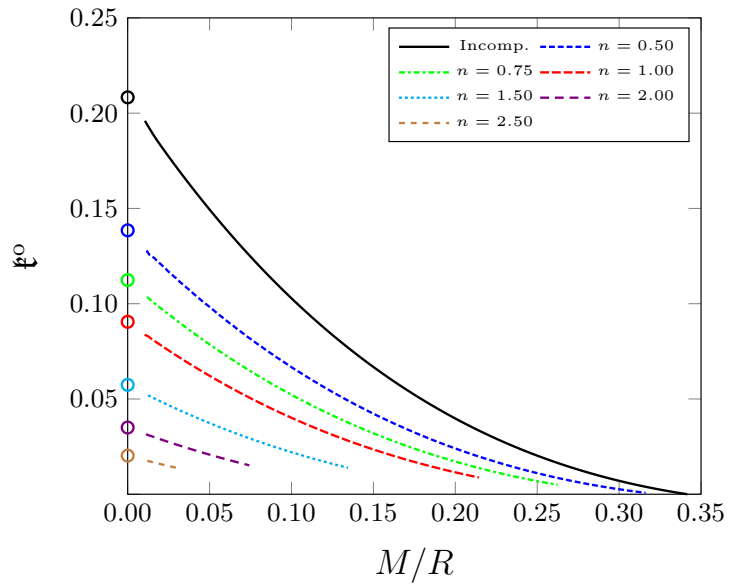

(d) Gravitomagnetic rotational-tidal Love number

FIG. 2: Love numbers $\left\{k_{2}^{\mathrm{el}}, k_{2}^{\mathrm{mag}}, \mathfrak{f}^{\mathrm{o}}, \mathfrak{k}^{\mathrm{o}}\right\}$ as a function of compactness $M / R$ for different polytropes and an incompressible fluid star. Circles indicate Love numbers calculated in the post-Newtonian approximation (see also Table IV].

$$
\delta \tilde{\lambda}_{M}^{(32)}=\frac{96}{\sqrt{5 \pi}} \mathfrak{F}^{\mathrm{o}}, \quad \delta \tilde{\lambda}_{E}^{(32)}=-144 \sqrt{\frac{7}{5}} \mathfrak{K}^{\mathrm{o}} .
$$

Provided our model of the NS's fluid interior is the same, our Love numbers can be compared with this mapping. Whereas in general the two frameworks make different assumptions about the state of the fluid-irrotational versus static - these states coincide in the gravitoelectric octupole sector when the star is free of $r$ - and $g$-modes [10, 28. Thus, for the same EoS, both sets of results for $\mathfrak{F}^{\circ}$ should agree. Pani, Gualtieri and Ferrari computed Love numbers for an $n=1$ energy polytrope with $\operatorname{EoS} p(\mu)=K \mu^{1+1 / n}$, which should coincide with our mass polytrope in the weakfield limit $M / R \rightarrow 0$. Nevertheless, Fig. 3 shows disagreement between our results and those of Ref. [26]. The fact that our general-relativistic results agree in the limit with the post-Newtonian calculation of $\mathfrak{f}^{\circ}$ gives us confidence in the conclusions we draw from our work, and simultaneously raises some questions about the computations of Ref. [26], at least at low compactness.

\section{LOVE NUMBERS FOR REALISTIC EQUATIONS OF STATE}

Having checked our method on polytropes, we now turn to realistic EoSs. We use a piecewise polytrope approximation to the tabulated EoSs computed in nuclear theory. In the stellar core, each model consists of a three-piece 


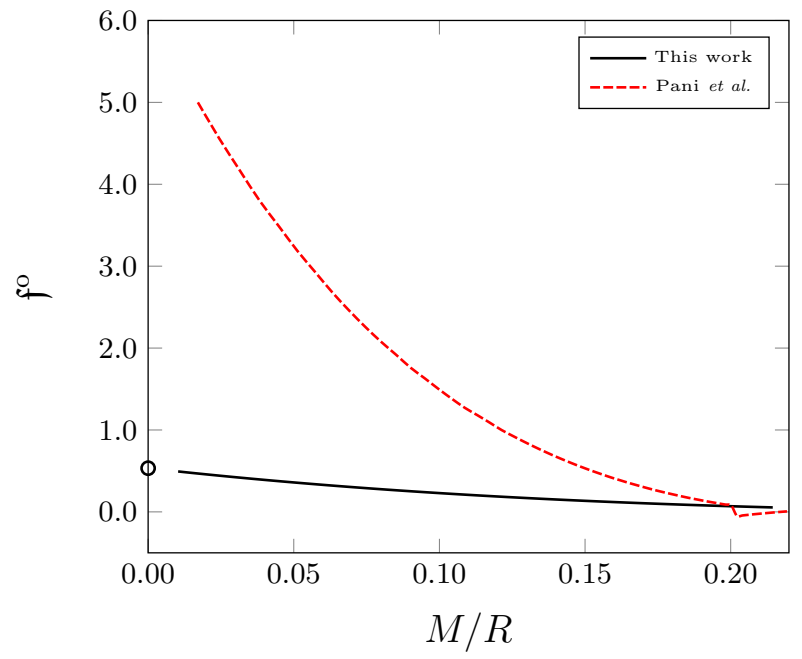

FIG. 3: Gravitoelectric rotational-tidal Love number $\mathfrak{f}^{\circ}$ for an $n=1$ polytrope. The solid black curve corresponds to our mass-polytrope results, whereas the dashed red curve is inferred via Eq. 25) from the energy-polytrope results labelled "POLYn1" in the bottom right panel of Fig. 5 of Ref. [26]. The black circle shows the result of the post-Newtonian calculation, which both mass and energy polytropes should match.

polytrope with an overall scale, resulting in four EoS-dependent parameters in total. The crust is always described by a four-piece polytropic approximation to the SLy EoS. This description of realistic EoSs follows closely that of Ref. 34, which we summarize here.

The stellar core EoS is taken to be

$$
p(\rho)= \begin{cases}K_{1} \rho^{\Gamma_{1}}, & \rho_{0} \leq \rho \leq \rho_{1} \\ K_{2} \rho^{\Gamma_{2}}, & \rho_{1} \leq \rho \leq \rho_{2} \\ K_{3} \rho^{\Gamma_{3}}, & \rho_{2} \leq \rho,\end{cases}
$$

with the transition rest mass densities $\rho_{1} \equiv 10^{14.7} \mathrm{~g} / \mathrm{cm}^{3}$ and $\rho_{2} \equiv 10^{15.0} \mathrm{~g} / \mathrm{cm}^{3}$. (The transition density $\rho_{0}$ is determined by matching at the crust-core interface.) The parameters $K_{i}$ are determined in terms of the overall scale of $p(\rho)$ by requiring continuity. Indeed, evaluating $p\left(\rho_{1}\right)$ sets $K_{1}=p\left(\rho_{1}\right) \rho_{1}^{-\Gamma_{1}}$, and imposing continuity at the interfaces $\rho_{1}$ and $\rho_{2}$ gives the recursive formula

$$
K_{i+1}=K_{i} \rho_{i}^{\Gamma_{i}-\Gamma_{i+1}}, \quad i=1,2 .
$$

Thus, the four parameters $\left\{p\left(\rho_{1}\right), \Gamma_{1}, \Gamma_{2}, \Gamma_{3}\right\}$ specify the EoS in the stellar core. Using continuity, the internal energy density $\epsilon$ is determined by the first law (5) to be

$$
\epsilon\left(\rho ; \rho_{i-1} \leq \rho \leq \rho_{i}\right)=a_{i} \rho+\frac{1}{\Gamma_{i}-1} K_{i} \rho^{\Gamma_{i}}, \quad \text { with } \quad a_{i}=\frac{\epsilon\left(\rho_{i-1}\right)}{\rho_{i-1}}-\frac{K_{i}}{\Gamma_{i}-1} \rho_{i-1}^{\Gamma_{i}-1}, \quad i=1,2,3,
$$

and from this, we obtain the the total energy density $\mu$. Since $a_{i}$ depends on $\epsilon\left(\rho_{i-1}\right), a_{1}$ is determined from the crust-core interface, and then $a_{2}$ and $a_{3}$ follow recursively.

The stellar crust model is similar to the core, except: (1) It consists of four polytropic phases - indexed by $i=-3,-2,-1,0$-instead of three; (2) Instead of $p\left(\rho_{-3}\right)$, we are directly given the constants $\left\{K_{-3}, K_{-2}, K_{-1}, K_{0}\right\}$; and (3) The crust model depends on the core via the interface density,

$$
\rho_{0}=\left(\frac{K_{1}}{K_{0}}\right)^{1 /\left(\Gamma_{0}-\Gamma_{1}\right)} .
$$

The remaining parameter needed to describe the crust, $a_{-3}$, is fixed to zero by requiring that $\epsilon / \rho \rightarrow 0$ in the zero rest mass density limit. Numerical parameters for the SLy crust model are provided in Ref. [34, and we display them in Table V] Given the core, the crust is fully specified, so the full stellar EoS is determined by the parameters $\left\{p\left(\rho_{1}\right), \Gamma_{1}, \Gamma_{2}, \Gamma_{3}\right\}$. 


\begin{tabular}{cccc}
\hline Phase & $K_{i}$ & $\Gamma_{i}$ & $\rho_{i}\left(\mathrm{~g} / \mathrm{cm}^{3}\right)$ \\
\hline-3 & $6.11252 \times 10^{12}$ & 1.58425 & $2.44034 \times 10^{7}$ \\
-2 & $9.54352 \times 10^{14}$ & 1.28733 & $3.78358 \times 10^{11}$ \\
-1 & $4.78764 \times 10^{22}$ & 0.62223 & $2.62780 \times 10^{12}$ \\
0 & $3.59389 \times 10^{13}$ & 1.35692 & $\rho_{0}$ \\
\hline \hline
\end{tabular}

TABLE V: Parameters of the four-piece polytropic crust model. The constants $K_{i}$ are in cgs units so that $p=K_{i} \rho^{\Gamma_{i}}$ is in dyne $/ \mathrm{cm}^{2}$. The crust-core interface density $\rho_{0}$ depends on the core parameters $K_{1}$ and $\Gamma_{1}$ through Eq. 29 .

\begin{tabular}{crrrrrr}
\hline \hline EoS & SLy & ENG & MPA1 & MS1 & MS1b & H4 \\
\hline $\log _{10} p\left(\rho_{1}\right)$ & 34.384 & 34.437 & 34.495 & 34.858 & 34.855 & 34.669 \\
$\Gamma_{1}$ & 3.005 & 3.514 & 3.446 & 3.224 & 3.456 & 2.909 \\
$\Gamma_{2}$ & 2.988 & 3.130 & 3.572 & 3.033 & 3.011 & 2.246 \\
$\Gamma_{3}$ & 2.851 & 3.168 & 2.887 & 1.325 & 1.425 & 2.144 \\
\hline \hline
\end{tabular}

TABLE VI: Parameters for the piecewise polytropic fits modelling the EoSs that we consider. In this table, $p$ is measured in units of dyne $/ \mathrm{cm}^{2}$.

Of the 34 candidate EoSs studied in Ref. [34, we consider the seven that give rise to stable stars with maximum masses greater than $2 M_{\odot}$ while avoiding superluminal sound propagation. This is consistent with the highest observed NS masses of $(1.97 \pm 0.04) M_{\odot}$ [35] and $(2.01 \pm 0.04) M_{\odot}$ [36. Five of the EoSs describe pure npe $\mu$ nuclear matterSLy [50], ENG [51], MPA1 [52, MS1 [53], and MS1b (which is identical to MS1 but with a low symmetry energy of $25 \mathrm{MeV}$ 34). The other two, H4 54 and ALF2 55, include nonstandard nuclear components (hyperons and color-flavor-locked quark matter, respectively). Parameters for the piecewise polytrope approximations are given in Table VI.

We restrict our attention to configurations with masses of astrophysical relevance $\left(M>M_{\odot}\right.$ [56, 57] that are stable against radial perturbations according to the turning point criterion. In addition, the crust in realistic NSs should constitute a small fraction of the whole star [58. Defining $r_{c c}$ as the radial position of the crust-core interface, and $M_{c r} \equiv M-m\left(r_{c c}\right)$ as the crust's mass, we note that our configurations satisfy

$$
\begin{aligned}
M_{c r} & <0.045 M, \\
R-r_{c c} & <0.15 R .
\end{aligned}
$$

In Fig. 4 we plot the Love numbers $\left\{k_{2}^{\mathrm{el}}, k_{2}^{\mathrm{mag}}, \mathfrak{f}^{\mathrm{o}}, \mathfrak{k}^{\circ}\right\}$ for our seven EoSs as functions of the stellar compactness. For comparison, we include Love numbers for $n=0.5, n=0.75$, and $n=1$ polytropes. We also display the Love numbers corresponding to a canonical $1.4 M_{\odot}$ NS in Table VII

A number of interesting features are apparent in Fig. 4 . First, all the Love numbers have a definite sign; we do not observe the zero-crossings reported in Ref. [26]. Second, the Love numbers for realistic EoSs are clustered between the $n=0.5$ and $n=1$ polytrope curves. There is, however, a qualitative difference between the slope of the curves for the npe $\mu$-matter EoSs (SLy, ENG, MPA1, MS1, MS1b; shown solid in Fig. 4) and the remaining two (H4, ALF2; shown dashed). Love numbers for the $n p e \mu$-matter EoSs also approach the $n=0.5$ polytrope curve at high

\begin{tabular}{cccccc}
\hline \hline EoS & $M / R$ & $k_{2}^{\text {el }}$ & $k_{2}^{\text {mag }}\left(\times 10^{-3}\right)$ & $\mathfrak{f}^{\circ}$ & $\mathfrak{k}^{\circ}\left(\times 10^{-2}\right)$ \\
\hline SLy & 0.1766 & 0.07617 & -5.977 & 0.1308 & 2.048 \\
ENG & 0.1727 & 0.08530 & -6.273 & 0.1457 & 2.272 \\
MPA1 & 0.1662 & 0.09130 & -6.341 & 0.1559 & 2.435 \\
MS1 & 0.1385 & 0.10544 & -6.179 & 0.1845 & 2.967 \\
MS1b & 0.1423 & 0.10714 & -6.328 & 0.1860 & 2.968 \\
H4 & 0.1482 & 0.09162 & -5.930 & 0.1611 & 2.604 \\
ALF2 & 0.1627 & 0.09777 & -6.522 & 0.1672 & 2.619 \\
\hline \hline
\end{tabular}

TABLE VII: Love numbers corresponding to a $1.4 M_{\odot}$ NS for each realistic EoS studied in Sec. V The star's compactness $M / R$ is also listed. 


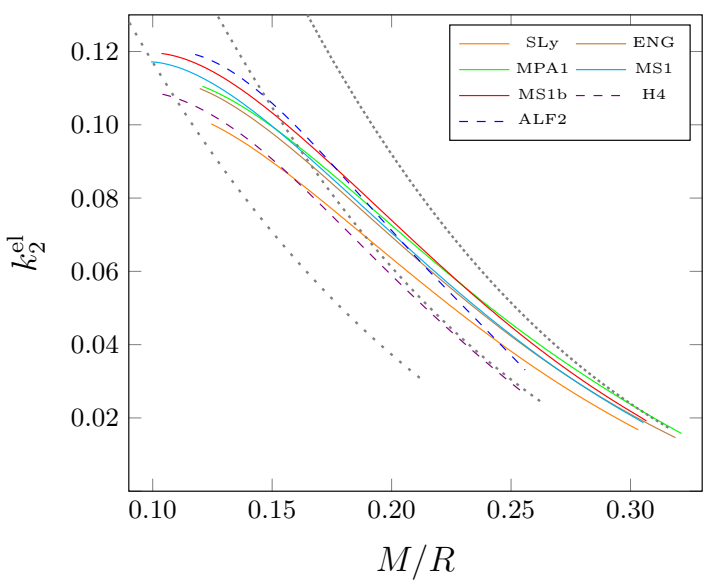

(a) Gravitoelectric tidal Love number

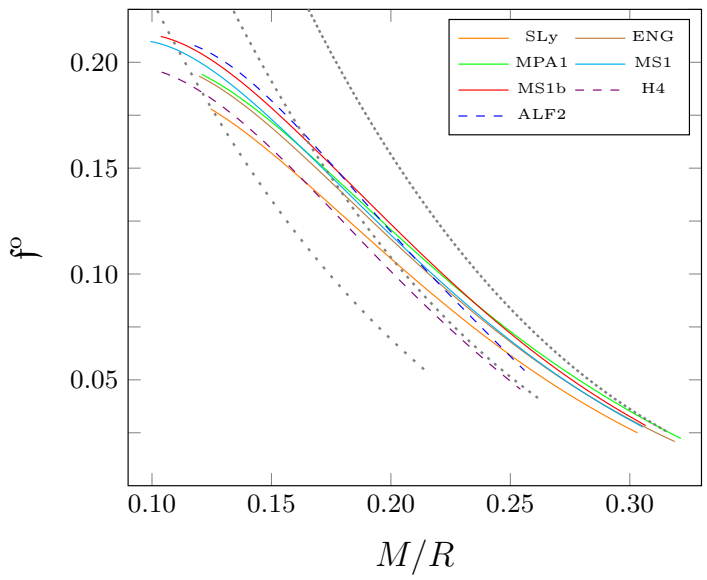

(c) Gravitoelectric rotational-tidal Love number

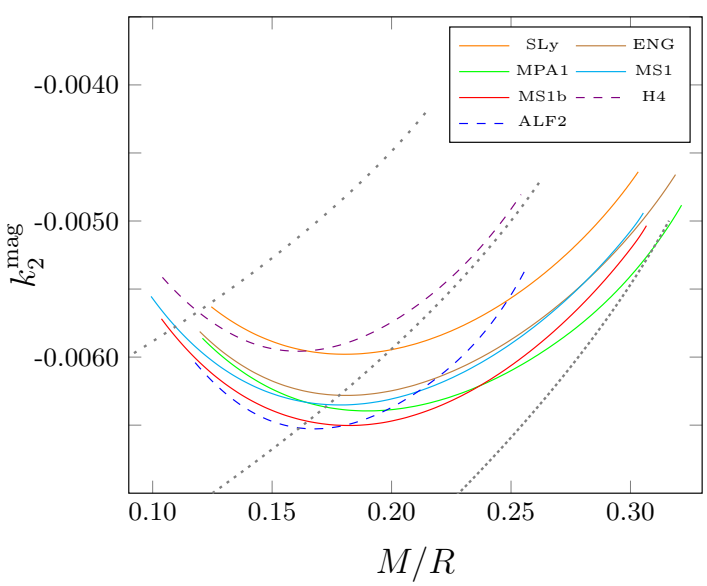

(b) Gravitomagnetic tidal Love number

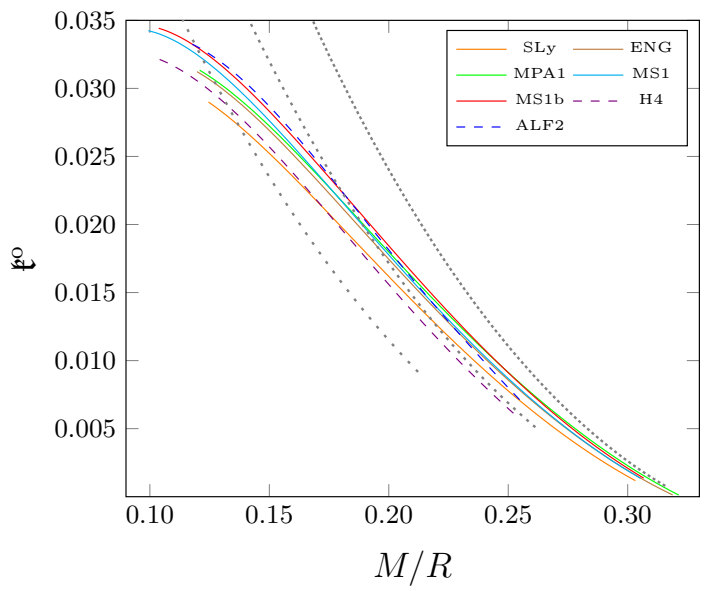

(d) Gravitomagnetic rotational-tidal Love number

FIG. 4: Love numbers for realistic-EoS NS models. Solid lines are used for $n p e \mu$-matter EoSs, while dashed lines denote exotic-matter EoSs. For comparison, we plot results for $n=0.5, n=0.75$ and $n=1$ polytropes with dotted lines (with more densely-spaced dots corresponding to smaller $n$ ).

compactness. This limiting behaviour is expected for SLy, ENG and MPA1, since - according to Table VI - those EoSs have adiabatic indices $\Gamma_{3}$ close to 3 (i.e. $n \approx 0.5$ ).

The limiting behavior also demonstrates that the crust makes a negligible contribution to the Love numbers at large compactness, since its adabiatic indices $\Gamma_{i}$ are significantly smaller than 3 . At low compactness, however, the crust becomes important because it constitutes a larger fraction of the NS, since the crust-core interface density $\rho_{0}$ is attained deeper inside the star. The softness of the crust is responsible for the flattening of the Love number curves observed for $M / R \lesssim 0.15$.

\section{I-LOVE RELATIONS}

As we described in the introduction, universal relations between macroscopic properties of NSs have emerged as a promising tool for observational astrophysics and gravitational-wave astronomy. To date, these relations have implicated the NS moment of inertia, the scaled tidal Love numbers, and the spin-induced quadrupole moment. Here, we extend the universal I-Love relations to include also the scaled rotational-tidal Love numbers.

We seek EoS-independent functional relationships between the dimensionless moment of inertia $\bar{I} \equiv I / M^{3}$ and each of the scaled Love numbers $\mathfrak{L} \in\left\{K_{2}^{\text {el }}, K_{2}^{\text {mag }}, \mathfrak{F}^{\circ}, \mathfrak{K}^{\circ}\right\}$. By plotting $\bar{I}$ against $\mathfrak{L}$ for every EoS in our sample $\xi^{4}$ as in

\footnotetext{
4 There is no established convention in the literature regarding whether $\bar{I}$ or $\mathfrak{L}$ should be taken as the independent variable; for instance,
} 


\begin{tabular}{crrrr}
\hline \hline Coefficient & $\bar{I}-K_{2}^{\text {el }}$ & $\bar{I}-K_{2}^{\text {mag }}$ & $\bar{I}-\mathfrak{F}^{\circ}$ & $\bar{I}-\mathfrak{K}^{\circ}$ \\
\hline$c_{0}$ & $8.115 \times 10^{-1}$ & 1.232 & $7.782 \times 10^{-1}$ & $9.325 \times 10^{-1}$ \\
$c_{1}$ & $1.763 \times 10^{-1}$ & $4.242 \times 10^{-1}$ & $1.518 \times 10^{-1}$ & $2.035 \times 10^{-1}$ \\
$c_{2}$ & $3.995 \times 10^{-2}$ & $2.635 \times 10^{-2}$ & $3.849 \times 10^{-2}$ & $4.147 \times 10^{-2}$ \\
$c_{3}$ & $-9.958 \times 10^{-4}$ & $-3.934 \times 10^{-3}$ & $7.846 \times 10^{-3}$ & $-7.056 \times 10^{-3}$ \\
$c_{4}$ & $-3.734 \times 10^{-3}$ & $-1.171 \times 10^{-4}$ & $-4.380 \times 10^{-3}$ & $-1.255 \times 10^{-3}$ \\
$c_{5}$ & $1.005 \times 10^{-3}$ & $-3.914 \times 10^{-3}$ & $-6.483 \times 10^{-3}$ & $1.292 \times 10^{-3}$ \\
$c_{6}$ & $9.147 \times 10^{-4}$ & $2.898 \times 10^{-3}$ & $7.131 \times 10^{-3}$ & $3.647 \times 10^{-5}$ \\
$c_{7}$ & $-7.091 \times 10^{-4}$ & $3.935 \times 10^{-4}$ & $-3.014 \times 10^{-3}$ & $-1.413 \times 10^{-4}$ \\
$c_{8}$ & $2.018 \times 10^{-4}$ & $-7.399 \times 10^{-4}$ & $6.544 \times 10^{-4}$ & $8.501 \times 10^{-6}$ \\
$c_{9}$ & $-2.674 \times 10^{-5}$ & $1.219 \times 10^{-4}$ & $-7.247 \times 10^{-5}$ & $6.347 \times 10^{-6}$ \\
$c_{10}$ & $1.377 \times 10^{-6}$ & $1.010 \times 10^{-5}$ & $3.249 \times 10^{-6}$ & $-8.400 \times 10^{-7}$ \\
\hline \hline
\end{tabular}

TABLE VIII: Coefficients of the $\bar{I}-\mathfrak{L}$ fit for each scaled Love number.

Fig. 5 and performing a $\log$-log polynomial fit

$$
\log _{10} \bar{I}_{\text {fit }}=\sum_{n=0}^{10} c_{n}\left(\log _{10} \mathfrak{L}\right)^{n}
$$

we can assess a given relation's degree of universality through the deviations

$$
\Delta(\%)=\frac{\left|\bar{I}-\bar{I}_{\text {fit }}\right|}{\bar{I}_{\text {fit }}} \times 100
$$

from the fit. The coefficients $c_{n}$ of our fits are listed in Table VIII, and the deviations are plotted in the insets of Fig. 5 .

We observe deviations from the universal $\bar{I}-K_{2}^{\text {el }}$ relation of less than $0.7 \%$, and of $0.1 \%$ on average, for our sample of EoSs. This is broadly consistent with the results reported in the literature 26, 30, which have maximum deviations of order $1 \%$. For the $\bar{I}-K_{2}^{\mathrm{mag}}$ fit, we find maximum deviations of $1.1 \%$ (average deviations of $0.3 \%$ ); residuals of less than $5 \%$ (2\% on average) were reported in Ref. [41] for NSs in the irrotational fluid state. The universality is weaker for the static fluid state: Refs. [26] and [41] observed maximum deviations of $6 \%$ and $10 \%$, respectively, in this case. We attribute our slightly smaller deviations overall to differences in the sample of EoSs used.

We find that the extended I-Love relations involving the scaled rotational-tidal Love numbers are also nearly EoSindependent, although the degree of universality is slightly weaker than for the scaled tidal Love numbers. For the $\bar{I}-\mathfrak{F}^{\circ}$ fit, deviations from universality average to $0.3 \%$, with a maximum of $1.3 \%$, whereas for the $\bar{I}-\mathfrak{K}^{\circ}$ fit they average to $0.6 \%$, and are always smaller than $2.5 \%$. These results are in sharp contrast to Ref. 26, which reported that I-Love universality was broken by the scaled rotational-tidal Love numbers - deviations of order $200 \%$ and $50 \%$ were found for $\delta \tilde{\lambda}_{M}^{(32)}$ (our $\mathfrak{F}^{\circ}$ ) and $\delta \tilde{\lambda}_{E}^{(32)}$ (our $\mathfrak{K}^{\circ}$ ), respectively, for NSs in the static state. We suspect that a discrepancy of this magnitude is not simply due to the difference in fluid state, and is likely rooted in the same problem that caused our disagreement for polytropes.

We observe that for the scaled tidal Love numbers, I-Love universality is weaker in the gravitomagnetic sector, as claimed by Ref. [26, and we see that the trend persists for the scaled rotational-tidal Love numbers. We also checked that for polytropes, softer EoSs depart more strongly from the realistic EoS fit (not shown in Fig. 5), extending existing intuition from the case of the scaled tidal Love numbers 30. Indeed, for polytropes with $n=0.5, n=0.75$, and $n=1$, we get deviations from the $\bar{I}-\mathfrak{F}^{\circ}$ fit of less than $1.4 \%, 1.75 \%$, and $3.7 \%$, respectively; for the $\bar{I}-\mathfrak{K}^{\circ}$ fit, the deviations are less than $2.7 \%, 3.1 \%$, and $6.0 \%$. We note that the degree of universality is again stronger in the gravitoelectric sector.

As a consequence of the extended I-Love relations, we also expect Love-Love universal relations to hold between each pair of scaled Love numbers, generalizing the universal $K_{2}^{\mathrm{mag}}-K_{2}^{\mathrm{el}}$ relation [40. We check these relations in Fig. 6

In Fig. 6 a we find that $K_{2}^{\mathrm{mag}}-K_{2}^{\mathrm{el}}$ universality holds to within $1.8 \%$ ( $0.6 \%$ on average) for NSs in the irrotational state. Refs. [26] and [40] found corresponding deviations of less than $3 \%$ and 10\%, respectively, but those results

Ref. 30] plots $\bar{I}$ in terms of $K_{2}^{\mathrm{el}}$, but Refs. 26, 41 plot the reverse. We choose to adopt the former arrangement here. This should be kept in mind as we compare the numerical values of the I-Love deviations with the latter references. 


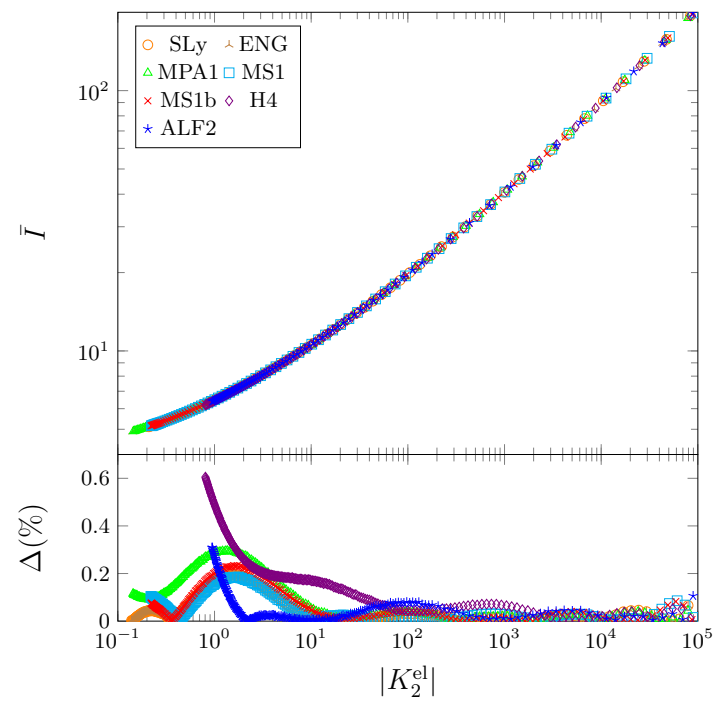

(a) $\bar{I}-K_{2}^{\text {el }}$ relation

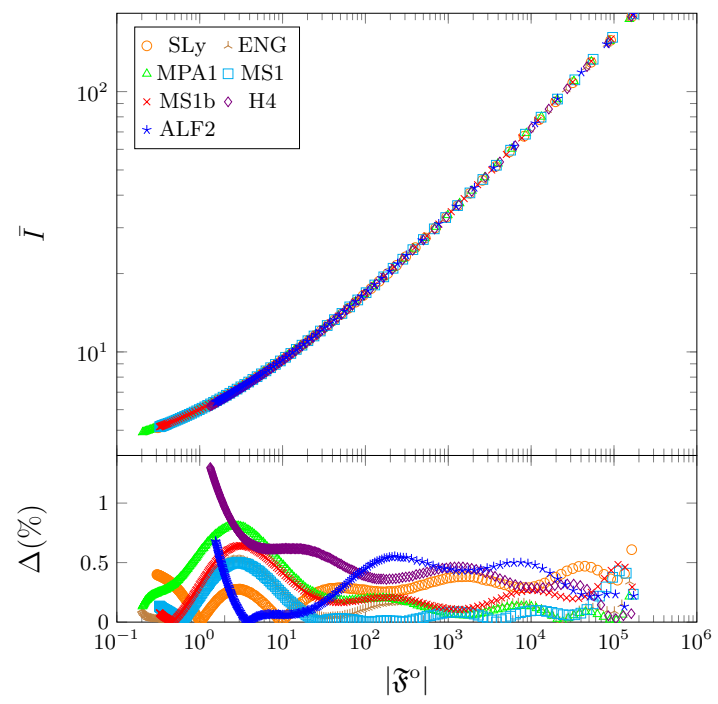

(c) $\bar{I}-\mathfrak{F}^{\circ}$ relation

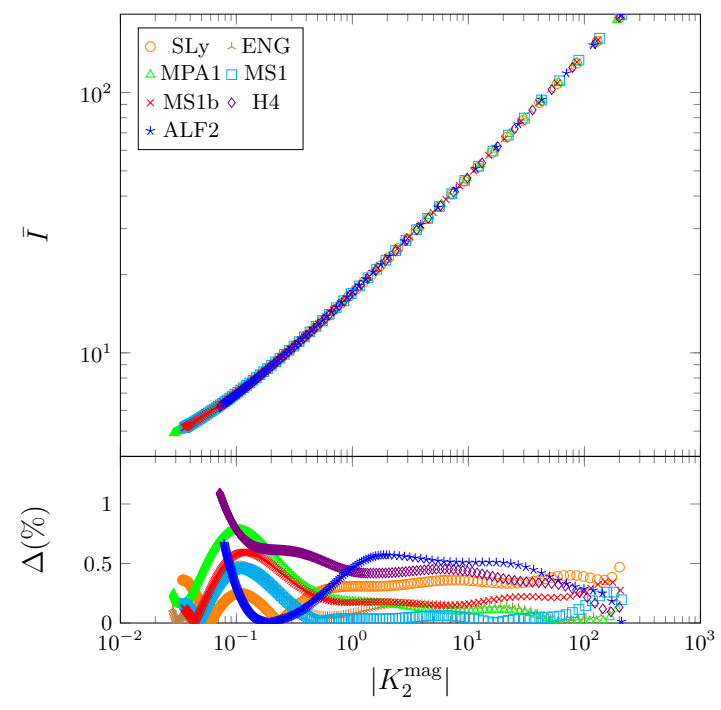

(b) $\bar{I}-K_{2}^{\operatorname{mag}}$ relation

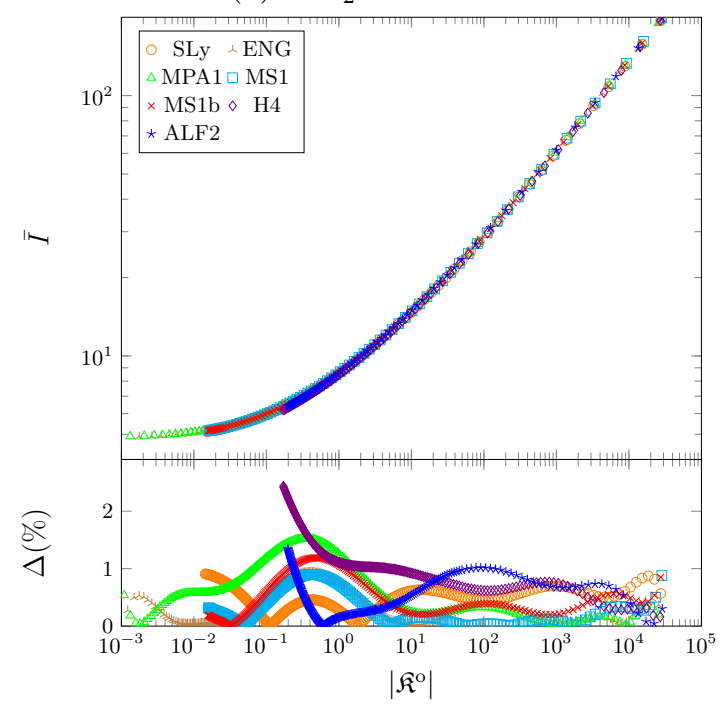

(d) $\bar{I}-\mathfrak{K}^{\circ}$ relation

FIG. 5: I-Love relations for the scaled Love numbers $\left\{K_{2}^{\mathrm{el}}, K_{2}^{\mathrm{mag}}, \mathfrak{F}^{\mathrm{o}}, \mathfrak{K}^{\circ}\right\}$ calculated with the seven realistic EoS models described in Sec. V. Note that the astrophysical range of interest corresponds to $4<\bar{I}<30$, but we include larger values of $\bar{I}$ for comparison with other analyses. Insets show the deviations $\Delta$ from universality (in \%) for each EoS with respect to fits of the form of Eq. (31) with the coefficients of Table VIII.

refer to the static fluid state. Relations between $K_{2}^{\mathrm{el}}$ and the scaled rotational-tidal Love numbers (Figs. 6b and 6c) are slightly less universal, with deviations of less than $4.5 \%$ (1\% on average) seen in the $\mathfrak{F}^{\circ}-K_{2}^{\text {el }}$ fit, and maximum deviations of $10.5 \%$ (2.5\% on average) for the $K_{2}^{\text {el }}-\mathfrak{K}^{\circ}$ fit 5 Similar relations involving $K_{2}^{\text {mag }}$ (Figs. 6d and 6e show a slightly better universality, with deviations of less than $1.2 \%(0.2 \%$ on average $)$ seen in the $\mathfrak{F}^{\circ}-K_{2}^{\text {mag }}$ fit, and maximum deviations of $4.3 \%$ ( $0.9 \%$ on average) for the $K_{2}^{\text {mag }}-\mathfrak{K}^{\circ}$ fit. Finally, the $\mathfrak{F}^{\circ}-\mathfrak{K}^{\circ}$ relation (Fig. 6f) shows maximum deviations of $6.9 \%$ (1.6\% on average). Coefficients for each of the fits are given in Table IX

Given that approximate universality holds between the dimensionless moment of inertia $\bar{I}$ and each of the scaled Love numbers $\left\{K_{2}^{\mathrm{el}}, K_{2}^{\mathrm{mag}}, \mathfrak{F}^{\mathrm{o}}, \mathfrak{K}^{\circ}\right\}$, we see no reason to expect it to be broken when other scaled rotational-tidal Love numbers are included. In particular, it may be worthwhile to revisit Ref. [26]'s claim that scaled quadrupole rotationaltidal Love numbers $\mathfrak{F}^{\mathfrak{q}}$ and $\mathfrak{K}^{\mathrm{q}}$ arising from couplings between the NS spin and an applied octupolar tidal field do not

\footnotetext{
5 The fact that $\mathfrak{K}^{\circ}$ approaches zero at large values of the compactness (see Fig. 4 is problematic for a log-log fit. We overcome this issue by taking $\mathfrak{K}^{\mathrm{O}}$ to be the independent variable for the fits in which it is involved.
} 


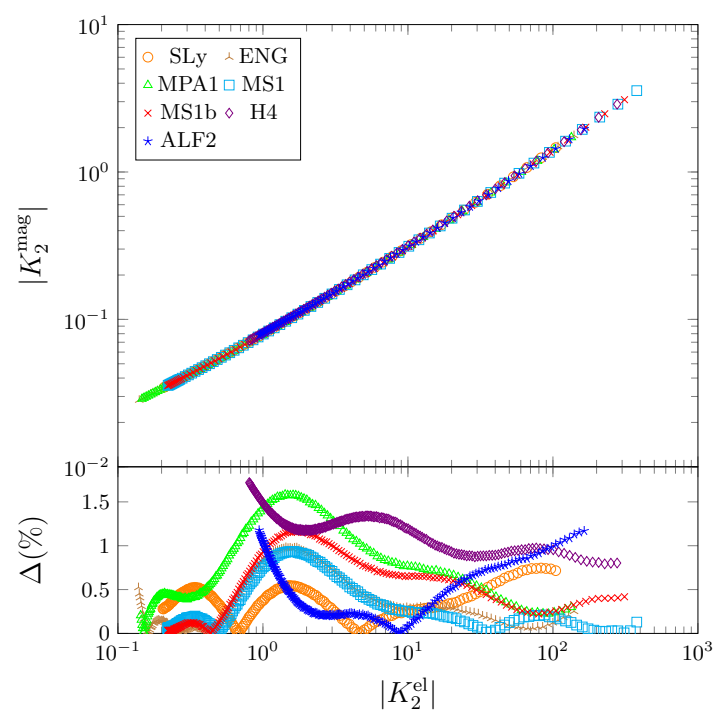

(a) $K_{2}^{\text {mag }}-K_{2}^{\text {el }}$ relation

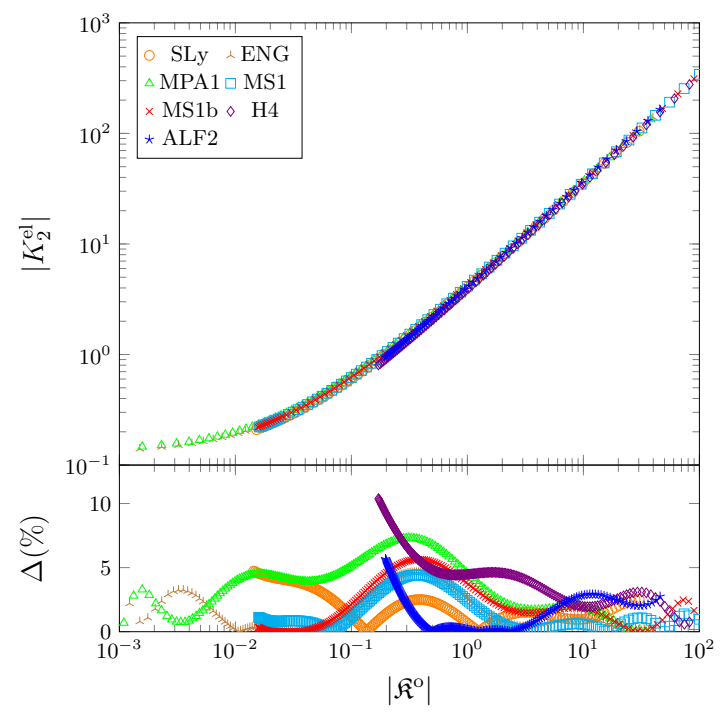

(c) $K_{2}^{\mathrm{el}-\mathfrak{K}^{\mathrm{o}}}$ relation

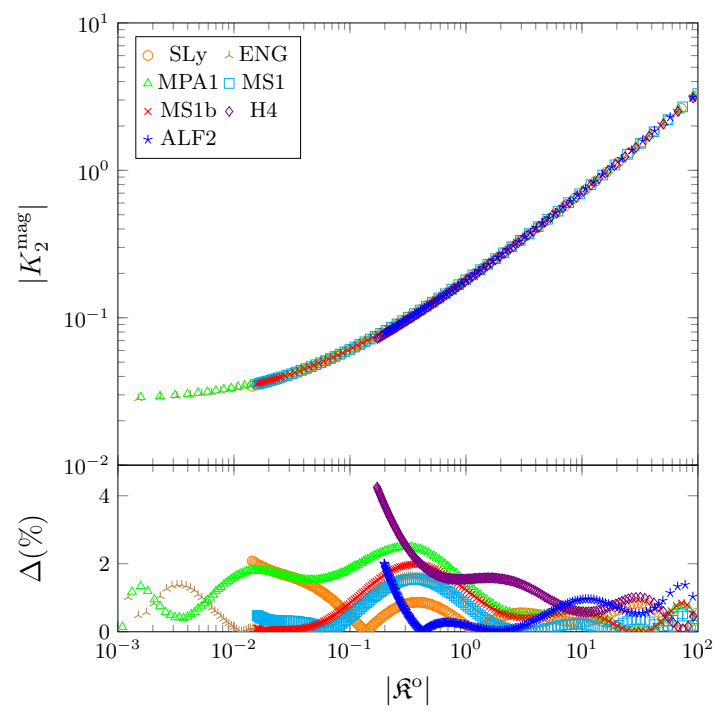

(e) $K_{2}^{\text {mag }} \mathfrak{K}^{\mathrm{o}}$ relation

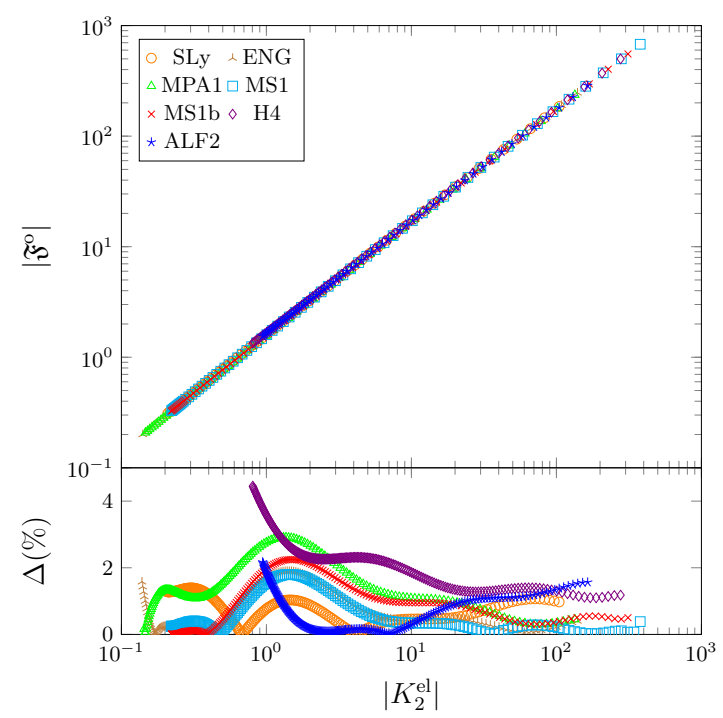

(b) $\mathfrak{F}^{\mathrm{o}}-K_{2}^{\text {el }}$ relation

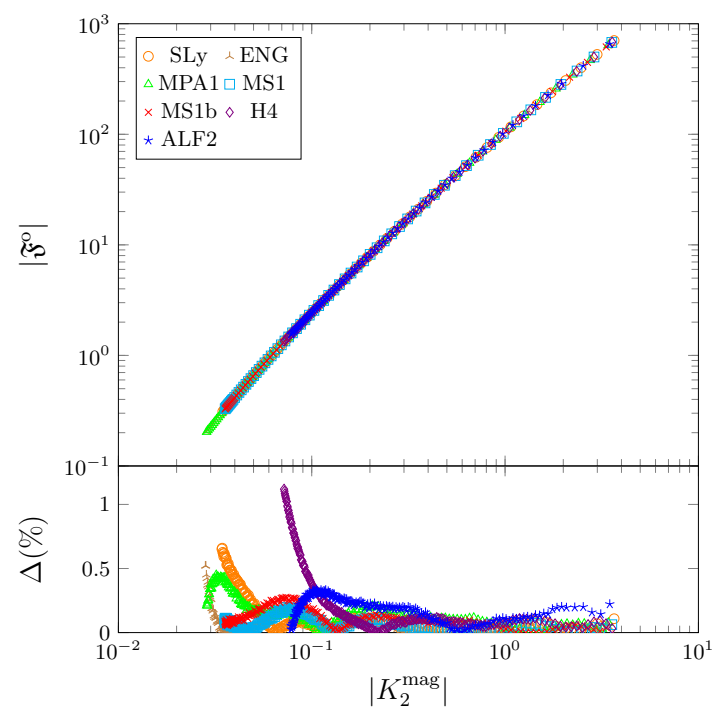

(d) $\mathfrak{F}^{\mathrm{o}}-K_{2}^{\mathrm{mag}}$ relation

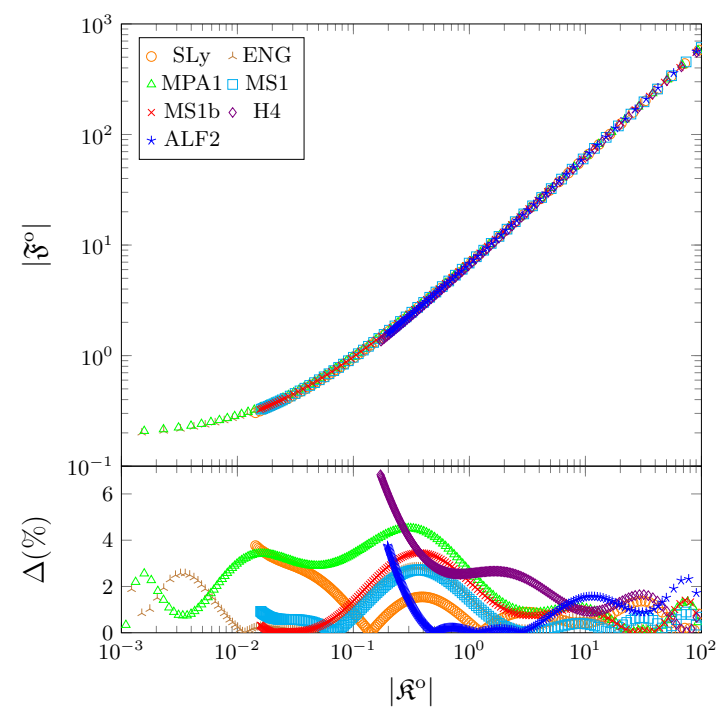

(f) $\mathfrak{F}^{\circ}-\mathfrak{K}^{\circ}$ relation

FIG. 6: Universal relations between each pair of scaled Love numbers. Insets show the deviations $\Delta$ from universality (in \%) for each EoS with respect to fits of the form of Eq. (31) with the coefficients of Table IX 


\begin{tabular}{|c|c|c|c|}
\hline Coefficient & $K_{2}^{\mathrm{mag}}-K_{2}^{\mathrm{el}}$ & $\mathfrak{F}^{\mathrm{o}}-K_{2}^{\mathrm{el}}$ & $K_{2}^{\mathrm{el}-\mathfrak{K}^{\mathrm{o}}}$ \\
\hline$c_{0}$ & -1.098 & $2.083 \times 10^{-1}$ & $6.083 \times 10^{-1}$ \\
\hline$c_{1}$ & $5.644 \times 10^{-1}$ & 1.050 & $9.359 \times 10^{-1}$ \\
\hline$c_{2}$ & $2.089 \times 10^{-2}$ & $-5.389 \times 10^{-2}$ & $5.264 \times 10^{-2}$ \\
\hline$c_{3}$ & $-1.826 \times 10^{-2}$ & $-2.082 \times 10^{-2}$ & $-8.223 \times 10^{-2}$ \\
\hline$c_{4}$ & $4.358 \times 10^{-2}$ & $9.608 \times 10^{-2}$ & $1.131 \times 10^{-2}$ \\
\hline$c_{5}$ & $-4.877 \times 10^{-3}$ & $-2.342 \times 10^{-2}$ & $4.248 \times 10^{-2}$ \\
\hline$c_{6}$ & $-3.331 \times 10^{-2}$ & $-7.012 \times 10^{-2}$ & $1.054 \times 10^{-3}$ \\
\hline$c_{7}$ & $2.430 \times 10^{-2}$ & $6.092 \times 10^{-2}$ & $-1.276 \times 10^{-2}$ \\
\hline$c_{8}$ & $-5.740 \times 10^{-3}$ & $-1.850 \times 10^{-2}$ & $-1.818 \times 10^{-3}$ \\
\hline$c_{9}$ & $3.740 \times 10^{-5}$ & $1.712 \times 10^{-3}$ & $1.345 \times 10^{-3}$ \\
\hline$c_{10}$ & $1.078 \times 10^{-4}$ & $7.841 \times 10^{-5}$ & $3.018 \times 10^{-4}$ \\
\hline Coefficient & $K_{2}^{\mathrm{mag}}-\mathfrak{K}^{\mathrm{o}}$ & $\mathfrak{F}^{\mathrm{O}}-\mathfrak{K}^{\mathrm{O}}$ & $\mathfrak{F}^{\mathrm{o}}-K_{2}^{\mathrm{mag}}$ \\
\hline$c_{0}$ & $-7.455 \times 10^{-1}$ & $8.324 \times 10^{-1}$ & 2.022 \\
\hline$c_{1}$ & $5.566 \times 10^{-1}$ & $9.474 \times 10^{-1}$ & 1.511 \\
\hline$c_{2}$ & $5.909 \times 10^{-2}$ & $4.750 \times 10^{-2}$ & $-9.236 \times 10^{-2}$ \\
\hline$c_{3}$ & $-3.377 \times 10^{-2}$ & $-5.985 \times 10^{-2}$ & $-5.106 \times 10^{-3}$ \\
\hline$c_{4}$ & $4.361 \times 10^{-3}$ & $9.396 \times 10^{-3}$ & $-2.805 \times 10^{-2}$ \\
\hline$c_{5}$ & $1.585 \times 10^{-2}$ & $2.685 \times 10^{-2}$ & $1.619 \times 10^{-1}$ \\
\hline$c_{6}$ & $2.551 \times 10^{-4}$ & $1.023 \times 10^{-3}$ & $2.206 \times 10^{-1}$ \\
\hline$c_{7}$ & $-4.713 \times 10^{-3}$ & $-8.216 \times 10^{-3}$ & $-1.670 \times 10^{-1}$ \\
\hline$c_{8}$ & $-6.642 \times 10^{-4}$ & $-1.275 \times 10^{-3}$ & $-4.769 \times 10^{-1}$ \\
\hline$c_{9}$ & $4.981 \times 10^{-4}$ & $8.753 \times 10^{-4}$ & $-3.085 \times 10^{-1}$ \\
\hline$c_{10}$ & $1.118 \times 10^{-4}$ & $2.021 \times 10^{-4}$ & $-6.576 \times 10^{-2}$ \\
\hline
\end{tabular}

TABLE IX: Coefficients for fits of the same form as Eq. 31) between each pair of scaled Love numbers $\mathfrak{L}_{1}-\mathfrak{L}_{2}$, with $\mathfrak{L}_{2}$ used as the independent variable. As noted in Footnote 5 , $\mathfrak{K}^{\circ}$ is used as the independent variable when it appears.

satisfy universal I-Love relations. We conjecture that all of Sec. II]s corrections to the leading-order gravitoelectric quadrupole tides are approximately expressible in terms of a single scaled Love number, for example $K_{2}^{\text {el }}$.

Finally, we remark that the original I-Love-Q study [30 also covered relations involving the rotational quadrupole moment $Q$; we do not investigate these relations here since $Q$ is second order in spin, and our perturbative calculation is limited to linear order. Nevertheless, we expect universal Love-Q relations for scaled rotational-tidal Love numbers to follow from the original I-Q relations combined with the extended I-Love relations presented here.

\section{DISCUSSION}

In this paper, we studied the Love numbers of slowly rotating NSs deformed by weak quadrupolar tides. We computed the rotational-tidal Love numbers $\mathfrak{f}^{\circ}$ and $\mathfrak{k}^{\circ}$ - for the first time for realistic NSs in the irrotational fluid state - as a function of the stellar compactness for seven chosen EoSs. For astrophysically relevant NS models, they lie in the ranges $0.03 \lesssim \mathfrak{f}^{\circ} \lesssim 0.22$ and $0.001 \lesssim \mathfrak{k}^{\circ} \lesssim 0.035$. To assist in future estimates of spin-corrections to tidal effects in NS binaries, we also provided the Love numbers' specific numerical values for canonical NSs in Table VII.

We showed that $\mathfrak{F}^{\circ}$ and $\mathfrak{K}^{\circ}$ satisfy extended I-Love and Love-Love relations that are universal to within a few percent in almost every case, in contradiction to previous work [26]. Despite a different choice of fluid state for the NS, we compared our results with those of Ref. 26] in the regime of overlap, and found that they do not agree there. This shows that the discrepancy in our conclusions is not simply a consequence of the choice of fluid state. As a check on our computations, we compared our general-relativistic results for polytropes to Love numbers we computed in post-Newtonian theory, and we showed that they agree in the weak-field limit. We also established that our polytrope Love numbers tend to those of an incompressible fluid as the stiffness of the EoS is increased. This gives us confidence in our conclusions.

Universality relations tell us that certain seemingly unrelated NS properties are in fact functionally interdependent. In this paper, we have shown that the set of interrelated quantities involved in I-Love universality can be extended to include $\mathfrak{F}^{\circ}$ and $\mathfrak{K}^{\circ}$. This extended I-Love universality supports the idea that - at an approximate level-NSs are described by just one more parameter than black holes, regardless of the EoS [59. This simplicity in the description of NSs is thought to be related to the emergence of an approximate symmetry in compact stars [37, but a complete the- 
oretical explanation remains elusive. It would be interesting to study universality relations involving quadrupole [26] and higher- $\ell$ scaled rotational-tidal Love numbers to see if universality holds.

The additional reduction in the parameter space of NSs brought about by extended I-Love universality is especially useful for gravitational-wave astronomy. When spin-coupled tidal effects are eventually incorporated into waveforms, no new parameters will be needed; to within the accuracy of measurements, it will suffice to use universality to replace the scaled rotational-tidal Love numbers with $K_{2}^{\mathrm{el}}$, the most easily measured Love number. Moreover, should a rotational-tidal Love number's contribution to the phasing of the waveform be degenerate with another effectsomething like the spin-spin and rotational quadrupole degeneracy [30, but at higher post-Newtonian order-an independent measurement of $I$ (from electromagnetic observations, for instance) or $K_{2}^{\mathrm{el}}$ (from gravitational wave measurements) could break the degeneracy.

As the rotational-tidal Love numbers have now been computed for a variety of candidate NS models, the next task is to determine their effect on the phase of the inspiral waveform. Based on their scalings in the metric, the rotational-tidal deformations can be just as large as the pure gravitomagnetic ones. Although it is unlikely that this precise hierarchy of sizes will carry over directly to the tidal phasing, rotational-tidal effects may well become important for future-generation detectors. Modeling spin corrections to the tidal phase is thus timely and important work, and the results presented here will be essential for that goal.

\section{ACKNOWLEDGMENTS}

We are glad to thank Eric Poisson for his insightful advice about this paper. We thank Luis Lehner, Kent Yagi, Justin Vines, and Peter Zimmerman for helpful discussions, and we acknowledge Paolo Pani for providing data for comparison. J. G. B. would like to thank the Perimeter Institute for full support via the Undergraduate Student Program. This work was supported in part by the Natural Sciences and Engineering Research Council of Canada. Research at the Perimeter Institute is supported by the Government of Canada through Industry Canada and by the Province of Ontario through the Ministry of Research and Innovation.

\section{Appendix A: Post-Newtonian Love numbers}

In this section, we calculate the Love numbers $k_{2}^{\mathrm{el}}, k_{2}^{\mathrm{mag}}, \mathfrak{f}^{\mathrm{o}}$, and $\mathfrak{k}^{\mathrm{o}}$ in post-Newtonian theory. The post-Newtonian results are expected to agree with the general-relativistic Love numbers in the zero-compactness limit. General weakfield formulas for the tidal Love numbers have been derived in the literature: in this regime, the gravitoelectric tidal Love number $k_{2}^{\mathrm{el}}$ reduces to the Newtonian Love number $k_{2}$ [8], which can be calculated with the recipe presented in Sec. 2.4 of Ref. 60; ; and the post-Newtonian gravitomagnetic tidal Love number $k_{2}^{\text {mag }}$ can be computed via the integral

$$
k_{2}^{\mathrm{mag}}=-\frac{2 \pi}{15 M R^{4}} \int_{0}^{R} \rho r^{6} d r .
$$

derived by Landry and Poisson in Ref. [10, 6

The case of the rotational-tidal Love numbers $\mathfrak{f}^{\circ}$ and $\mathfrak{k}^{\circ}$ has received an incomplete treatment in the literature, and we address it more fully in Secs. A 1 and A 2 below. We derive formulas that are valid for arbitrary barotropic EoSs, including the incompressible fluid model. We also find that $k_{2}$ is automatically determined as a byproduct of the integration of Eq. A6 required to compute $\mathfrak{f}^{\circ}$. Throughout this appendix, we restore the physical units of Newton's constant $G$ and the speed of light $c$.

\section{Post-Newtonian $\mathfrak{f}^{\circ}$}

As a nonlinear phenomenon, the rotational-tidal couplings which give rise to fo and $\mathfrak{k}^{\circ}$ are absent in Newtonian theory. Accordingly, a post-Newtonian analysis is required to determine the zero-compactness values of these Love numbers. In this section, we focus on the gravitoelectric rotational-tidal Love number $\mathfrak{f}^{\circ}$. The only post-Newtonian

\footnotetext{
${ }^{6}$ In terms of notation, $k_{2}^{\text {mag }}[$ here $]=\tilde{k}_{2}^{\text {mag }}[\mathrm{LP}]$. We set their parameter $\lambda$ to 1 , which represents the irrotational state that we study here. The fluid quantities in Eq. A1] are solutions to the Newtonian equations of structure, Eq. A3.
} 
calculation of $\mathfrak{f}^{\circ}$ in the literature was performed by Landry and Poisson in Ref. 27. for the special case of an incompressible fluid; they found that

$$
\mathfrak{f}^{\mathrm{o}}[\mathrm{LP}]=-\left(\frac{2 G M}{c^{2} R}\right)^{5} \mathfrak{F}^{\mathrm{o}}[\mathrm{LP}]=\frac{75}{56} \approx 1.33929 \quad \text { (incompressible fluid) }
$$

in the weak-field regime - we recall that they employ a different definition of $\mathfrak{f}^{\circ}$ than we do [cf. Eq. (23)]. However, Ref. [27]'s result disagrees with our general-relativistic calculation of $\mathfrak{f}^{\mathrm{o}}[\mathrm{LP}]$, as is clear from Fig. 1. This discrepancy originates from a term that was missed by Landry and Poisson, as we demonstrate below. (One may note that Eq. A2 gives the post-Newtonian value of $\mathfrak{f}^{\mathrm{O}}[\mathrm{here}]$; indeed, the term missed in Ref. [27] happens to correspond precisely to the difference between the definitions of $\mathfrak{f}^{\mathrm{o}}\left[\right.$ here] and $\mathfrak{f}^{\mathrm{o}}[\mathrm{LP}]$.)

We perform a completely general post-Newtonian calculation of $\mathfrak{f}^{\circ}$ for an arbitrary barotropic EoS. Our strategy is as follows: first, we take the unperturbed configuration to be a nonrotating, isolated Newtonian star. Next, we introduce rotational and $\ell=2$ tidal perturbations. We solve the problem at linear order in the perturbations using Newtonian theory, and then calculate the leading-order terms in the post-Newtonian metric describing the spacetime outside the tidally deformed, rotating star 7 Finally, we compare this post-Newtonian metric to the general-relativistic metric of Sec. IIIC in the weak-gravity limit, and we solve for $\mathfrak{f}^{\circ}$ algebraically. The remainder of this section is dedicated to presenting these manipulations in detail.

The density profile $\rho$ of a non-rotating, isolated Newtonian star is a solution to the equations of structure for a spherically symmetric ball of fluid, 60.

$$
\frac{d p}{d r}=-\rho \frac{G m}{r^{2}}, \quad \frac{d m}{d r}=4 \pi r^{2} \rho .
$$

These equations are supplemented by a barotropic $\operatorname{EoS} p=p(\rho)$. Once this background configuration is determined, a velocity field

$$
v^{a}=\epsilon_{a b c} \Omega^{b} x^{c}
$$

describing rigid rotation is imposed on the unperturbed star. We point out that the density perturbation caused by rotation is proportional to $\Omega^{2}$ because centrifugal effects are of that order. Therefore, to the approximation level of the general-relativistic perturbation theory, which neglects terms of second order in spin, we can ignore any rotational effects in the density perturbation.

The next step is to introduce the tidal field and to compute the density perturbation that distinguishes the tidally deformed, rotating star from the unperturbed configuration. Since we are only interested in the value of gravitoelectric rotational-tidal Love number $\mathfrak{f}^{\circ}$, which is associated with $\mathcal{E}_{a b}$, we turn off the tidal source's gravitomagnetic contribution by setting $\mathcal{B}_{a b}=0$. Because the gravitoelectric part of the tidal field is simply the usual Newtonian gravitational field, the density perturbation is therefore the same as in the Newtonian theory of linear tides - see, for instance, Sec. 2.4 of Ref. 60. However, Ref. [60] only solves the problem outside the star, while here we must solve the internal problem for the tidally induced density perturbation.

A pure tidal quadrupole field in Newtonian gravity has the gravitational potential

$$
U^{\mathrm{tid}}=-\frac{1}{2} \mathcal{E}_{a b} x^{a} x^{b}
$$

where $\mathcal{E}_{a b} \equiv-\partial_{a b} U^{\mathrm{ext}}(0)$ is a constant STF tensor comprising second derivatives (evaluated at the origin) of the total gravitational potential $U^{\text {ext }}$ produced by the tidal source. The deformation induced in response to $U^{\text {tid }}$ is measured by the well-known Newtonian Love number $k_{2}$, and we compute the tidal perturbations $\delta U$ and $\delta \rho$ of the body's gravitational potential and density in terms of this Love number. Because the perturbations are proportional to the applied tidal field, for purely quadrupolar linear tides we write $\delta U=\tilde{U}(r) \mathcal{E}^{\mathrm{q}}$, where $\mathcal{E}^{\mathrm{q}} \equiv \mathcal{E}_{a b} n^{a} n^{b}$ according to Table II Poisson's equation then implies that $\tilde{U}$ satisfies the ODE

$$
r^{2} \frac{d^{2} \tilde{U}}{d r^{2}}+2 r \frac{d \tilde{U}}{d r}-\left(6+\frac{4 \pi r^{4} \rho^{\prime}}{m}\right) \tilde{U}=-\frac{2 \pi r^{6} \rho^{\prime}}{m} .
$$

Outside the star, the solution to this equation is $\tilde{U}=-k_{2} R^{5} / r^{3}$ [60. In the interior, Eq. A6 must be solved numerically. A local analysis near $r=0$ reveals that $\tilde{U}^{\prime}(0)=\tilde{U}(0)=0$ is required for regularity. We therefore solve

\footnotetext{
7 The structure of the tidally deformed, rotating star need not be determined beyond Newtonian order precisely because we are only interested in the leading-order relativistic terms in the post-Newtonian metric. Solving for the structure at 1PN would only contribute higher-order relativistic terms. This discussion is complemented by Footnote 8
} 
Eq. (A6) using a shooting method, imposing the regularity conditions and the continuity of $\tilde{U}(r)$ and $\tilde{U}^{\prime}(r)$ across $r=R$. The solution automatically determines the value of the Newtonian Love number, $k_{2}=-\tilde{U}(R) / R^{2}$, as a consequence of the matching conditions at the stellar surface.

The density perturbation is given by Eq. (2.218) of Ref. [60], $\delta \rho=-\xi^{j} \partial_{j} \rho=-\xi^{r} \rho^{\prime}$, where the second equality follows from the spherical symmetry of the unperturbed configuration. The radial component of the Lagrangian displacement vector $\xi^{j}$ is determined to be

$$
\xi^{r}=\frac{r^{2}}{G m}\left(\tilde{U}-\frac{r^{2}}{2}\right) \mathcal{E}^{\mathrm{q}}
$$

via the perturbed Euler equation (see e.g. Eq. (2.214) of Ref. [60]). The density perturbation is therefore

$$
\delta \rho=-\frac{\rho^{\prime} r^{2}}{G m}\left(\tilde{U}-\frac{r^{2}}{2}\right) \mathcal{E}^{\mathrm{q}}
$$

With Eqs. (A4) and (A8), along with the radial density profile $\rho(r)$ obtained from Eq. (A3), the relevant part of the structure of the tidally deformed, rotating star is known up to order $\chi^{a} \mathcal{E}_{b c}$. We are now in a position to compute the contribution of the gravitoelectric rotational-tidal effects to the post-Newtonian metric outside the star. Since we are solely interested in the Love number $\mathfrak{f}^{\circ}$, which multiplies the bilinear potential $\mathcal{F}_{A}^{o}$ (defined in Table $\Pi$ modulo a hidden factor of $c^{2}$ ) in the general-relativistic metric of Eq. (10), we only keep track of the contributions proportional to $\mathcal{F}_{a}^{\mathrm{o}}$ (the Cartesian version of the potential; $\mathcal{F}_{A}^{\mathrm{o}}=\mathcal{F}_{a}^{\mathrm{o}} n_{A}^{a}$ ) in our calculation and we ignore all other terms. Moreover, it turns out that the gauge transformation required to properly compare the post-Newtonian and general-relativistic metrics does not affect the terms involved in the calculation of $\mathfrak{f}^{\circ}$.

To leading order, the post-Newtonian metric components are given in quasi-Cartesian coordinates by ${ }^{8}$

$$
g_{t t}=-1+\frac{2}{c^{2}} U+\mathcal{O}\left(c^{-4}\right), \quad g_{t a}=-\frac{4}{c^{3}} U_{a}+\mathcal{O}\left(c^{-5}\right), \quad g_{a b}=\delta_{a b}\left(1+\frac{2}{c^{2}} U\right)+\mathcal{O}\left(c^{-4}\right),
$$

where $U$ is the Newtonian gravitational potential and $U_{a}$ is a vector potential obeying the field equation $\nabla^{2} U_{a}=$ $-4 \pi G \rho v_{a}$. The tidally induced perturbations to this metric involve $\delta U_{a}$, the bilinear perturbation of the vector potential, which satisfies

$$
\nabla^{2} \delta U_{a}=-4 \pi G\left(\delta_{1} j_{a}+\delta_{2} j_{a}\right)
$$

with $\delta_{1} j_{a} \equiv \delta \rho v_{a}$ and $\delta_{2} j_{a} \equiv \rho \delta v_{a}$. Here, $\delta v_{a}$ is the tidal perturbation of the velocity field $v^{a}$. The solution to this equation is the sum of the contributions sourced by each $\delta_{i} j_{a}$, namely

$$
\delta_{i} U_{a}=G \int \frac{\delta_{i} j_{a}\left(\mathbf{x}^{\prime}\right)}{\left|\mathbf{x}-\mathbf{x}^{\prime}\right|} d^{3} x^{\prime}
$$

Only $\delta_{1} U_{a}$ gives a contribution proportional to $\mathcal{F}_{a}^{\circ}$, so $\delta v_{a}$ is irrelevant for our purposes. Following the techniques described in Ref. [27, which are crucial for solving Eq. [A11] analytically, we obtain

$$
\delta_{1} U_{a}=-\frac{4 \pi G c}{7} \frac{B}{r^{4}} \mathcal{F}_{a}^{o}, \quad \text { with } \quad B \equiv-\int_{0}^{\infty} \frac{\rho^{\prime} r^{8}}{m}\left(\tilde{U}-\frac{r^{2}}{2}\right) d r
$$

where we have left out any terms not proportional $\mathcal{F}_{a}^{\circ}$. Therefore, to leading order, the perturbation in the metric due to gravitoelectric rotational-tidal couplings is

$$
\delta g_{t a}=-\frac{4}{c^{3}} \delta U_{a}=\frac{16 \pi G}{7 c^{2}} \frac{B}{r^{4}} \mathcal{F}_{a}^{\mathrm{o}} .
$$

To compare this perturbation to the one appearing in the general-relativistic metric, we must re-express it in quasispherical coordinates; doing so, we obtain

$$
\delta g_{t A}=\delta g_{t a} x_{A}^{a}=\frac{16 \pi G}{7 c^{2}} \frac{B}{r^{3}} \mathcal{F}_{A}^{\mathrm{o}},
$$

\footnotetext{
${ }^{8}$ It can be seen here that post-Newtonian corrections to the structure, which are of order $c^{-2}$ according to the post-Newtonian Euler equation - see e.g. Eq. (8.119) in Ref. [60]-would add contributions of order $c^{-4}$ and $c^{-5}$ to the metric.
} 
where $x_{A}^{a}=\partial x^{a} / \partial \theta^{A}$. We emphasize that this equality leaves out terms not proportional to $\mathcal{F}_{A}^{\mathrm{o}}$. Comparing Eq. A14 to the $t A$ component of the general-relativistic external metric, Eq. [10, we infer that the radial function $f_{t}^{\mathrm{o}}(r)$ multiplying $\mathcal{F}_{A}^{\mathrm{o}}$ is

$$
f_{t}^{\mathrm{o}}[\mathrm{PN}]=\frac{16 \pi G}{7 c^{2}} \frac{B}{r^{3}}
$$

in post-Newtonian theory. To determine the Love number fo, we compare Eq. A15 to the general-relativistic expression for $f_{t}^{\circ}$, given in Table III] in the zero-compactness limit. To leading post-Newtonian order, we find

$$
f_{t}^{\mathrm{o}}(r)[\mathrm{GR}]=-\frac{4 G}{M c^{2}} \frac{I R^{5}}{r^{3}} \mathfrak{f}^{\mathrm{o}}
$$

where the moment of inertia $I$ can be calculated via the Newtonian formula

$$
I=\frac{8 \pi}{3} \int_{0}^{R} \rho r^{4} d r
$$

Equating expressions $\mathrm{A} 15$ and $\mathrm{A} 16$ we obtain the general expression

$$
\mathfrak{f}^{\mathrm{o}}=\frac{4 \pi}{7 I} \frac{M}{R^{5}} \int_{0}^{R} \frac{\rho^{\prime} r^{8}}{m}\left(\tilde{U}-\frac{r^{2}}{2}\right) d r
$$

for the post-Newtonian gravitoelectric rotational-tidal Love number. In practice, Eq. A18, along with Eqs. A3, (A6) and A17, must be integrated numerically for a given barotropic EoS ${ }^{9}$

In the special case of an incompressible fluid, the discontinuity of the density $\rho$ at the stellar surface induces a jump in the derivative of the potential $\tilde{U}$ at $r=R$, whose contribution must be taken into account when matching the internal and external versions of $\tilde{U}$. In order to evaluate this jump, we integrate Eq. A6 through the stellar surface as described in Appendix B, obtaining

$$
\frac{d \tilde{U}}{d r}\left(R^{+}\right)-\frac{d \tilde{U}}{d r}\left(R^{-}\right)=\frac{3}{R}\left[\frac{R^{2}}{2}-\tilde{U}(R)\right] .
$$

With this correction, the incompressible fluid Love number $\mathfrak{f}^{\mathrm{o}}$ can be calculated in post-Newtonian theory via the recipe presented above.

Finally, we identify a mistake made by the authors of Ref. [27] in their comparison of the post-Newtonian and general-relativistic versions of the metric: they asserted that their radial function $f_{4}^{\circ}$ reduces to $2\left(2 G M / c^{2} r\right)^{6} \mathfrak{F}^{\circ}[\mathrm{LP}]$ in the weak-field limit. However, it should really reduce to $\left(2 G M / c^{2} r\right)^{6}\left[2 \mathfrak{F}^{\mathrm{o}}[\mathrm{LP}]-(10 / 3) K_{2}^{\text {el }}\right]$. Including this missing term, their post-Newtonian result in Eq. A2 becomes $f^{\circ}[\mathrm{LP}] \approx 0.08929$, which agrees with our general-relativistic incompressible fluid results from Fig. 1 .

\section{Post-Newtonian $\mathfrak{k}^{\mathrm{O}}$}

A post-Newtonian calculation of gravitomagnetic rotational-tidal deformations was performed by Poisson and Douçot in Ref. 61. However, the authors were interested in effects other than the specific value of the gravitomagnetic rotational-tidal Love number $\mathfrak{k}^{\circ}$, and consequently they only computed $\mathfrak{k}^{\circ}$ for the special case of an $n=1$ polytrope. Here, we adapt their calculation to a general barotropic EoS. Our starting point is Eqs. (6.4), (6.5), (6.11b), and (7.9) of Ref. 61. The octupole perturbation $\delta U^{\ell=3}$ of the post-Newtonian gravitational potential can be decomposed in terms of the radial unit vector $n^{a}$ as

$$
\delta U^{\ell=3}=\mathrm{U}^{\mathrm{o}}(r) \mathcal{K}_{a b c} n^{a} n^{b} n^{c} .
$$

This equation involves the bilinear moment $\mathcal{K}_{a b c}$ defined in Table $\mathrm{I}$, along with the post-Newtonian version of the gravitomagnetic tidal quadrupole moment, $\mathcal{B}_{a b} \equiv 2 \epsilon^{c d}{ }_{(a} \partial_{b) c} U_{d}^{\text {ext }}(0)$. Here, $\partial_{b c} U_{a}^{\text {ext }}(0)$ denotes partial derivatives of

\footnotetext{
9 We note that an integration by parts can be performed in Eq. A18 to better condition it for numerical integration. Furthermore, this step allows one to treat the incompressible fluid in the same way as the other EoSs by eliminating the factor of $\rho^{\prime}$, which is singular at $r=R$ for a body of uniform density. In all cases, the boundary terms vanish unequivocally since the boundaries of integration originating from Eq. A12 extend over the whole spatial domain.
} 
the vector potential of the tidal source evaluated at the center of the star. The radial function $\mathrm{U}^{\circ}(r)$ has to satisfy Eq. (7.11) of Ref. 61,

$$
r^{2} \frac{d^{2} \mathrm{U}^{\mathrm{o}}}{d r^{2}}+2 r \frac{d \mathrm{U}^{\mathrm{o}}}{d r}-\left(12+\frac{4 \pi r^{4} \rho^{\prime}}{m}\right) \mathrm{U}^{\mathrm{o}}=\frac{8 \pi}{9 c^{2}} \frac{r^{7} \rho^{\prime}}{m}
$$

This equation is solved outside the star by setting the derivative $\rho^{\prime}$ to zero and picking out the solution decaying with $r$, which represents the body's tidal response. Doing so, we find that $\mathrm{U}^{\mathrm{o}}(r)=\alpha r^{-4}$ in the exterior, for $\alpha=$ constant. In the interior, Eq. A21 must be solved numerically. Local analysis at the center reveals that the regular solution has $\mathrm{U}^{\mathrm{o}}(r) \propto r^{3}$ for sufficiently small $r$. Equation A21 can therefore be solved using a shooting method with boundary conditions that match $\mathrm{U}^{\circ}(r)$ and its first derivative to the external solution at $r=R$. With the matching value $\mathrm{U}^{\circ}(R)$ at hand, Eq. A20 can be compared at $r=R$ to the octupole perturbation $\delta U_{\text {eff }}^{\ell=3}$ inferred from the general-relativistic external metric in the weak-field limit,

$$
\delta U_{\mathrm{eff}}^{\ell=3}=-\frac{2}{c^{2}}\left(\frac{2 G M}{c^{2}}\right)^{5} \mathfrak{K}^{\mathrm{o}} \frac{I}{M r^{4}} \mathcal{K}_{a b c} n^{a} n^{b} n^{c} ;
$$

cf. Eq. (A4) in Ref. 61]. Here, $I$ is the Newtonian moment of inertia. Equating the post-Newtonian and generalrelativistic perturbations, we solve algebraically for the post-Newtonian value of the Love number $\mathfrak{k}^{\circ}$ and find

$$
\mathfrak{k}^{\mathrm{O}}=\frac{M c^{2} \mathrm{U}^{\mathrm{o}}(R)}{2 I R}
$$

As in the calculation for $\mathfrak{f}^{\circ}$, the discontinuity of the incompressible fluid density distribution at the stellar surface induces a jump in the derivative of the potential $U^{\circ}$ at $r=R$. The jump is found by integrating Eq. A21 through the stellar surface as described in Appendix B this yields

$$
\frac{d \mathrm{U}^{\mathrm{o}}}{d r}\left(R^{+}\right)-\frac{d \mathrm{U}^{\mathrm{o}}}{d r}\left(R^{-}\right)=-\frac{3}{R}\left[\mathrm{U}^{\mathrm{o}}(R)+\frac{2 R^{3}}{9 c^{2}}\right] .
$$

With this correction, the incompressible fluid Love number $\mathfrak{k}^{\circ}$ can be calculated in post-Newtonian theory via the recipe presented above.

\section{Appendix B: Incompressible fluid model}

In this appendix, we adapt the recipe of Sec. III for computing the Love numbers to the case of an incompressible fluid. Though the model itself is unphysical, we expect its Love numbers to bound those of other barotropic EoSs from above ${ }^{10}$, one can view the incompressible fluid as the $n \rightarrow 0$ limit of the polytropic models.

In principle, one can employ the method described in Sec. IIIE to compute the Love numbers associated with any barotropic EoS. However, the implementation of this method turns out to require special care for an incompressible fluid. We examine the subtleties related to this case below; our treatment of the gravitoelectric tidal Love number is essentially the same as that of Refs. [7, 65, 66], and we take a similar approach in the novel case of the rotational-tidal Love numbers.

The incompressible fluid model has a constant rest mass density $\rho_{*}>0$ inside the star, which abruptly drops to zero outside. Hence, its density profile is $\rho(r)=\rho_{*} \Theta(R-r)$, where $\Theta$ denotes the Heaviside step function. In this case, the first law of thermodynamics written as $d \mu=h d \rho$, where $h$ is the fluid's enthalpy, implies that the total energy density $\mu$ is some constant $\mu_{*}$ in the interior of the star, so it can be expressed as

$$
\mu=\mu_{*} \Theta(R-r) .
$$

The total mass of the star is then $M=4 \pi \mu_{*} R^{3} / 3$ by Eq. (7). Evidently, the radial derivative of the total energy density is singular at the surface of the star. This implies that radial derivatives of other metric and fluid variables will be generically singular at $r=R$. In particular, some of the radial functions which appear in the metric ansatz Eq. 10 and are directly related to the Love numbers have a discontinuous first derivative at the surface.

10 The incompressible fluid does not provide the lowest upper bound on the Love numbers by any means. Finer upper bounds using the most extreme NS EoS 62] compatible with causality have been investigated in Refs. 63 64. 
Let us focus first on the gravitoelectric sector. In order to compute $K_{2}^{\text {el }}$ and $\mathfrak{F}^{\mathrm{o}}$ using the method described in Sec. IIIE 1, we have to integrate Eqs. (16)- $\sqrt{18}$ in the interior of the star. The presence of the singular derivative $d \mu / d p$ in Eqs. (17) and (18) induces derivative discontinuities of the radial functions $e_{t t}^{\mathrm{q}}$ and $f_{t}^{\mathrm{o}}$ at the stellar surface, although the functions themselves remain continuous as required by the matching conditions on the spacetime at $r=R$. Thus, corrections due to such discontinuities need to be taken into account in the integration of $e_{t t}^{\mathrm{q}}$ and $f_{t}^{o}$. In order to evaluate these discontinuities, we integrate Eqs. 17) and (18) from $R^{-} \equiv R-\varepsilon$ to $R^{+} \equiv R+\varepsilon$, where $\varepsilon>0$, and we take the limit $\varepsilon \rightarrow 0$. In both cases, we get non-vanishing integrals involving terms of the form $\zeta(r)(\mu+p)(d \mu / d p)$ for some radial function $\zeta(r)$. Differentiating Eq. (B1) and using the TOV equation, Eq. (9), we find

$$
\int_{R^{-}}^{R^{+}} \zeta(r)(\mu+p) \frac{d \mu}{d p} d r=\int_{R^{-}}^{R^{+}} \zeta(r) r^{2} f \frac{\mu_{*} \delta(r-R)}{m+4 \pi r^{3} p} d r=\zeta(R) R^{2} f(R) \mu_{*} / M .
$$

With the latter result at hand, we obtain the derivative jumps

$$
\begin{aligned}
\frac{d e_{t t}^{\mathrm{q}}}{d r}\left(R^{+}\right)-\frac{d e_{t t}^{\mathrm{q}}}{d r}\left(R^{-}\right) & =-\frac{3}{R} e_{t t}^{\mathrm{q}}(R) \\
\frac{d f_{t}^{\mathrm{o}}}{d r}\left(R^{+}\right)-\frac{d f_{t}^{\mathrm{o}}}{d r}\left(R^{-}\right) & =-3 R e^{-2 \psi(R)}[\omega(R)+1] e_{t t}^{\mathrm{q}}(R)
\end{aligned}
$$

from Eqs. (17) and (18). For completeness, we note that the absence of the singular term $d \mu / d p$ in Eq. (16) prevents any discontinuity in $d \omega / d r$, and thus no jump correction needs to be incorporated in the integration of that equation.

Next, in order to compute the scaled gravitomagnetic Love numbers $K_{2}^{\text {mag }}$ and $\mathfrak{K}^{\text {o }}$ following the method described in Sec. III E 2, we need to integrate Eqs. (19)-(21) in the interior of the star. As in the case of Eq. (16), no correction is needed in the integration of Eq. 19p. However, in principle, one has to account for derivative jumps in the functions $k_{t r 1}^{\mathrm{o}}$ and $k_{t t}^{\mathrm{o}}$. We evaluate these jumps by integrating Eqs. 20$)$ and $(21)$ from $R^{-}$to $R^{+}$, taking the limit $\varepsilon \rightarrow 0$. The new singular terms are either of the form

$$
\zeta(r) \frac{d \mu}{d p} k_{t r 1}^{\mathrm{o}} \quad \text { or } \quad \zeta(r) \frac{d \mu}{d p} \frac{d k_{t r 1}^{\mathrm{o}}}{d r} .
$$

In order to evaluate their integrals across the stellar surface, we recall the fact that $k_{t r 1}^{\mathrm{o}}=0$ for $r \geq R$, so we can always replace $k_{t r 1}^{o}$ by $k_{t r 1}^{o} \Theta(R-r)$ independently of its explicit functional form in the interior. The same argument applies to the derivative $d k_{t r 1}^{\mathrm{o}} / d r$ and the pressure $p$, so that we can also write $d k_{t r 1}^{\mathrm{o}} / d r=\left(d k_{t r 1}^{\mathrm{o}} / d r\right) \Theta(R-r)$ and $\mu+p=\left(\mu_{*}+p\right) \Theta(R-r)$. This technical artifice leads to cancellations of Heaviside functions in the singular terms. Thus, differentiation of Eq. (B1) and substitution of $d p / d r$ from Eq. (9) lead to

$$
\int_{R^{-}}^{R^{+}} \zeta(r) \frac{d \mu}{d p} k_{t r 1}^{\mathrm{o}} d r=\int_{R^{-}}^{R^{+}} \zeta(r) r^{2} f \frac{\mu_{*} \delta(r-R)}{\left(\mu_{*}+p\right)\left(m+4 \pi r^{3} p\right)} k_{t r 1}^{\mathrm{o}} d r=\zeta(R) R^{2} f(R) k_{t r 1}^{\mathrm{o}}(R) / M
$$

and likewise for the integral of the singular term involving the derivative $d k_{t r 1}^{\mathrm{o}} / d r$. By virtue of the continuity of the function $k_{t r 1}^{\mathrm{o}}$ in $r=R$, and the fact that $k_{t r 1}^{\mathrm{o}}(R)=0$, from Eq. 20 we obtain the derivative jump

$$
\frac{d k_{t r 1}^{\mathrm{o}}}{d r}\left(R^{+}\right)-\frac{d k_{t r 1}^{\mathrm{o}}}{d r}\left(R^{-}\right)=-\int_{R^{-}}^{R^{+}} \delta(r-R) \frac{d k_{t r 1}^{\mathrm{o}}}{d r} d r .
$$

From the exterior solution we know that $d k_{t r 1}^{\mathrm{o}} / d r=0$ at $r=R$; thus, continuity of $d k_{t r 1}^{\mathrm{o}} / d r$ across the surface is sufficient to satisfy Eq. B7). We therefore conclude that no jump corrections are needed in the integration of Eq. 20. Similarly, when Eq. 21 is integrated across the surface, we get the derivative discontinuity

$$
\frac{d k_{t t}^{\mathrm{o}}}{d r}\left(R^{+}\right)-\frac{d k_{t t}^{\mathrm{o}}}{d r}\left(R^{-}\right)=\frac{1}{R}\left[2(2 \omega(R)-3) b_{t}^{\mathrm{q}}(R)-3 k_{t t}^{\mathrm{o}}(R)\right]
$$

With the addition of these jump corrections, the set of Love numbers $\left\{K_{2}^{\text {el }}, K_{2}^{\text {mag }}, \mathfrak{F}^{\text {o }}, \mathfrak{K}^{\circ}\right\}$ can be computed for an incompressible fluid by following the method described in Sec. IIIE.

\section{Appendix C: Quadrupole rotational-tidal Love numbers}

Two kinds of scaled quadrupole rotational-tidal Love numbers are alluded to in this paper: the $\ell=2$ Love numbers that arise from couplings between the NS spin and the tidal quadrupole moments $\mathcal{E}_{a b}, \mathcal{B}_{a b}$, which we may call $\mathfrak{E}^{\mathrm{q}}$ and 
$\mathfrak{B}^{\mathrm{q}}$ following prior work $27+29$; and the $\ell=2$ Love numbers that are associated with couplings between the NS spin and the tidal octupole moments $\mathcal{E}_{a b c}, \mathcal{B}_{a b c}$, which we referred to as $\mathfrak{F}^{\mathrm{q}}$ and $\mathfrak{K}^{\mathrm{q}}$ in Sec. VI. The latter were studied by Pani, Gualtieri and Ferrari in Ref. [26], but were omitted in this work. The former were found by Landry [28] to possess a universal value of 1/120 for material bodies, independently of the EoS.

In this appendix, we clarify the nature of the scaled quadrupole rotational-tidal Love numbers $\mathfrak{E}^{\mathrm{q}}$, $\mathfrak{B}^{\mathrm{q}}$ generated by the external tidal quadrupole. The scaled Love number $\mathfrak{E}^{\mathrm{q}}$ is associated with the bilinear quadrupole moment $\hat{\mathcal{E}}_{a b} \equiv 2 \chi^{c} \epsilon_{c d(a} \mathcal{E}_{b)}^{d}$ introduced in Ref. [27]. This bilinear moment makes its appearance in the metric ansatz of Eq. 10] through the scalar potential $\hat{\mathcal{E}}^{\mathrm{q}} \equiv \hat{\mathcal{E}}_{a b} n^{a} n^{b}$ defined in Table II] According to Ref. [27], an equivalent expression for the potential is

$$
\hat{\mathcal{E}}^{\mathrm{q}}=-\chi \partial_{\phi} \mathcal{E}^{\mathrm{q}}
$$

Let us consider the effect on Eq. C1 of a shift

$$
\phi \rightarrow \phi-\kappa \chi
$$

in the angular coordinate $\phi$, where $\kappa$ is an arbitrary parameter. To first order in $\chi$, this corresponds to a change

$$
\mathcal{E}^{\mathrm{q}} \rightarrow \mathcal{E}^{\mathrm{q}}+\kappa \hat{\mathcal{E}}^{\mathrm{q}}
$$

in the tidal potential. The effect of this shift on the external metric ansatz, Eq. (4.4) of Ref. [27, is to take

$$
\begin{aligned}
\hat{e}_{t t}^{\mathrm{q}} & \rightarrow \hat{e}_{t t}^{\mathrm{q}}-\kappa e_{t t}^{\mathrm{q}}, \\
\hat{e}_{r r}^{\mathrm{q}} & \rightarrow \hat{e}_{r r}^{\mathrm{q}}-\kappa e_{r r}^{\mathrm{q}}, \\
\hat{e}^{\mathrm{q}} & \rightarrow \hat{e}^{\mathrm{q}}-\kappa e^{\mathrm{q}} .
\end{aligned}
$$

As can be seen from the expressions for the radial functions given in Table IV of Ref. [27], this amounts to a shift

$$
\mathfrak{E}^{\mathrm{q}} \rightarrow \mathfrak{E}^{\mathrm{q}}-\kappa K_{2}^{\mathrm{el}}
$$

in the Love number. Hence, $\mathfrak{E}^{\mathrm{q}}$ is gauge-dependent, and can be adjusted to make $\hat{e}_{t t}^{\mathrm{q}}, \hat{e}_{r r}^{\mathrm{q}}$ and $\hat{e}^{\mathrm{q}}$ vanish. In the Regge-Wheeler gauge, this is achieved by setting $\mathfrak{E}^{\mathrm{q}}=1 / 120$, as dictated by the interior solution [28].

The shift $\mathrm{C} 2$ also produces a change in $\hat{\mathcal{B}}_{A}^{\mathrm{q}} \equiv \epsilon_{a b c} n^{b} \hat{\mathcal{B}}_{d}^{c} n^{d} n_{A}^{a}=-\chi \partial_{\phi} \mathcal{B}_{A}^{\mathrm{q}}$, where the bilinear tidal moment is $\hat{\mathcal{B}}_{a b} \equiv 2 \chi^{c} \epsilon_{c d(a} \mathcal{B}^{d}{ }_{b)}$. The net effect is to shift the scaled Love number

$$
\mathfrak{B}^{\mathrm{q}} \rightarrow \mathfrak{B}^{\mathrm{q}}-\kappa K_{2}^{\mathrm{mag}}
$$

Once $\kappa$ has been selected to set $\mathfrak{E}^{\mathrm{q}}$ to a desired value, the gauge freedom in $\phi$ is exhausted, and $\mathfrak{B}^{\mathrm{q}}$ acquires a physical meaning: Landry has shown that it reflects the presence of an $r$-mode in the star.

Since we have demonstrated that the specific values of $\mathfrak{E}^{q}$ and $\mathfrak{B}^{q}$ are gauge-dependent, we conclude that they are not true scaled Love numbers. Rather, $\mathfrak{E}$ is a gauge constant associated with the freedom to shift the angular coordinate $\phi$; fixing this freedom sets the value of $\mathfrak{B}^{\mathfrak{q}}$ up to a residual physical dependence on internal $r$-modes. The point is that the couplings between $\chi^{a}$ and $\mathcal{E}_{a b}, \mathcal{B}_{a b}$ produce only two actual scaled rotational-tidal Love numbers, $\mathfrak{F}^{\circ}$ and $\mathfrak{K}^{\circ}$, not four as was claimed by Refs. 27.29. Finally, although this argument is formulated in the Regge-Wheeler gauge, we remark that a similar argument holds in the light-cone gauge [49] employed in Sec. III of Ref. [27].

\section{Appendix D: Comparison with Pani, Gualtieri \& Ferrari}

In this appendix, we derive the mappings between our scaled Love numbers $K_{2}^{\text {el }}, K_{2}^{\text {mag }}, \mathfrak{F}^{\mathrm{o}}, \mathfrak{K}^{\mathrm{o}}$ and their equivalents in Ref. [26]. A subset of these relations appeared without a detailed derivation in Ref. [28]. They permit us to compare our quantitative results with those of Ref. [26, as we do in Sec. IV].

Our Love numbers are defined as integration constants associated with decaying solutions in the exterior metric [27]. The Love numbers of Ref. 26] are defined in terms of derivatives of induced multipole moments with respect to tidal moments. Nonetheless, the expressions obtained by Pani, Gualtieri \& Ferrari ultimately involve metric components with integration constants of their own: their scaled tidal Love numbers are

$$
\tilde{\lambda}_{E}^{(2)}=\frac{2 \gamma_{2}}{\sqrt{5 \pi} \alpha_{2}}, \quad \tilde{\lambda}_{M}^{(2)}=\frac{\gamma_{2}^{*}}{480 \alpha_{2}^{*}}
$$


and their scaled rotational-tidal Love numbers are

$$
\delta \tilde{\lambda}_{M}^{(32)}=\frac{44 \sqrt{35} \gamma_{2}+21 \gamma_{32}}{28 \sqrt{7 \pi} \alpha_{2}}, \quad \delta \tilde{\lambda}_{E}^{(32)}=-\gamma_{32}^{*} / \alpha_{2}^{*} .
$$

The constants $\alpha_{2}, \alpha_{2}^{*}, \gamma_{2}, \gamma_{2}^{*}, \gamma_{32}, \gamma_{32}^{*}$ can be read off the perturbations (22)-(27) of Ref. [26]. Rather than relating our Love numbers explicitly to multipole moments, we choose to compare coordinate expressions for the metric and directly identify the constants appearing in Eqs. (D1)-(D2). In general, this would require a transformation of the metric, but fortunately both works employ Boyer-Lindquist $(t, r, \theta, \phi)$ coordinates and the Regge-Wheeler gauge.

The exterior solution of Ref. [26] is obtained by supplementing its background metric (9) with the perturbations (22)-(27) of that paper ${ }^{11}$ Because Pani, Gualtieri \& Ferrari restrict themselves to axisymmetric perturbations, we specialize our ansatz (10) to axisymmetry by dropping the terms with hatted potentials. We then compare the metric components order-by-order in a perturbative expansion, beginning with the background (which, for the purposes of this appendix, we take to include the rotation). A trivial identification of the coordinates, and the relations $e^{\nu} \rightarrow f$, $\mathcal{M} \rightarrow M, \omega \rightarrow \Omega(1-\omega)$, bring their background metric into the same form as ours.

The gravitoelectric tidal perturbations appear in the diagonal components of the metric. In Ref. [26], the $t t$ component of the perturbation is $\delta g_{t t}^{\mathrm{tid}}=e^{\nu} H_{0}^{(2)} Y^{20}$, where $Y^{\ell m}(\theta, \phi)$ denotes a spherical harmonic and

$$
H_{0}^{(2)}=\alpha_{2} y(y-2)+\gamma_{2}\left[-3+\frac{1}{2-y}-\frac{1}{y}+3 y+\frac{3}{2}(y-2) y \ln (1-2 y)\right]
$$

with $y \equiv r / M{ }^{12}$ Our corresponding metric perturbation is $\delta g_{t t}^{\mathrm{tid}}=e_{t t}^{\mathrm{q}} \mathcal{E}^{\mathrm{q}}$. The exterior solution for $e_{t t}^{\mathrm{q}}$ is given in Table III and we may expand the tidal potential in spherical harmonics as $\mathcal{E}^{\mathrm{q}}=\sum_{m} \mathcal{E}_{m}^{\mathrm{q}} Y^{\ell m}$ [27]. Setting the two perturbations equal, we find that

$$
\alpha_{2}=4 \sqrt{\frac{\pi}{5}} M^{2} \mathcal{E}_{0}^{\mathrm{q}}, \quad \gamma_{2}=-32 \sqrt{5 \pi} K_{2}^{\mathrm{el}} M^{2} \mathcal{E}_{0}^{\mathrm{q}} .
$$

These relations imply the mapping

$$
\tilde{\lambda}_{E}^{(2)}=-16 \sqrt{\frac{5}{\pi}} K_{2}^{\mathrm{el}}
$$

for the scaled gravitoelectric Love number. One can verify that with these associations the $r r$ and $A B$ components of the gravitoelectric tidal perturbation also match exactly.

The gravitoelectric rotational-tidal perturbation appears only in the $t A$ components of the metric. The octupole deformation is given as $\delta g_{t}^{\ell=3}=\delta h_{0}^{(3)} X_{A}^{30}$ in Ref. [26]; $X_{A}^{\ell m}=\left(-\partial_{\phi} Y^{\ell m} / \sin \theta, \sin \theta \partial_{\theta} Y^{\ell m}\right)$ is an odd-parity vector spherical harmonic and

$$
\begin{aligned}
\delta h_{0}^{(3)}= & -\frac{M \chi}{6720 y^{2}}\left\{-128 \sqrt{35} y(5 y-4) \alpha_{2}\right. \\
& -3\left[8 \sqrt{35}\left(16+44 y-90 y^{2}-270 y^{3}+945 y^{4}-405 y^{5}-y\left(64-80 y+1080 y^{3}-1350 y^{4}+405 y^{5}\right) \tanh { }^{-1} \frac{1}{1-y}\right) \gamma_{2}\right. \\
& \left.\left.+35 y\left(8+20 y+60 y^{2} 0-210 y^{3}+90 y^{4}+15 y^{3}\left(8-10 y^{2}+3 y^{2}\right) \ln (1-2 / y)\right) \gamma_{32}\right]\right\}
\end{aligned}
$$

Since $\alpha_{2}$ and $\gamma_{2}$ are known from Eq. (D4), $\gamma_{32}$ is the only undetermined parameter. Our equivalent metric perturbation is $\delta g_{t}^{\ell=3}=f_{t}^{\circ} \mathcal{F}_{A}^{\mathrm{o}}$, with $f_{t}^{\mathrm{o}}$ given in Table III and $\mathcal{F}_{A}^{\mathrm{o}}=\frac{1}{5} \chi \mathcal{E}_{0}^{\mathrm{q}} X_{A}^{30}$ in axisymmetry [27]. The comparison yields

11 Eqs. (22)-(27) of Ref. [26] are the components relevant for this discussion, but the full metric perturbation can be found in Appendix A of that paper. In practice, we make use of the perturbed metric provided as a MAтнемAтісA @ notebook in the Supplemental Material to Ref. [26] at http://link.aps.org/supplemental/10.1103/PhysRevD.92.124003

12 There appears to be a misprint in the sign of the second term in Eq. (22) in the published version of Ref. [26. The sign given here matches that found in the Supplemental Material. 


$$
\gamma_{32}=\frac{128}{5} \sqrt{\frac{\pi}{7}}\left(\frac{275}{3} K_{2}^{\mathrm{el}}+28 \mathfrak{F}^{\mathrm{o}}\right) M^{2} \mathcal{E}_{0}^{\mathrm{q}},
$$

and with this association the $\ell=3$ gravitoelectric rotational-tidal perturbations agree. It then follows that the scaled gravitoelectric rotational-tidal Love numbers are related by the first of the mappings in Eq. (25). This relation matches the one introduced in Ref. [28], after taking into account our redefinition of $\mathfrak{F}^{\circ}$.

We proceed in a similar fashion in the gravitomagnetic sector. The tidal perturbation appears exclusively in the $t A$ component of the metric, and equating our expression $\delta g_{t A}^{\mathrm{tid}}=b_{t}^{\mathrm{q}} \mathcal{B}_{A}^{\mathrm{q}}=b_{t}^{\mathrm{q}} \mathcal{B}_{0}^{\mathrm{q}} X_{A}^{20}$ with that of Ref. [26], $\delta g_{t A}^{\mathrm{tid}}=h_{0}^{(2)} X_{A}^{20}$, we identify

$$
\alpha_{2}^{*}=-\frac{4}{3} \sqrt{\frac{\pi}{5}} M^{2} \mathcal{B}_{0}^{\mathrm{q}}, \quad \gamma_{2}^{*}=-128 \sqrt{5 \pi} K_{2}^{\operatorname{mag}} M^{2} \mathcal{B}_{0}^{\mathrm{q}}
$$

These relations imply that the scaled gravitomagnetic Love number $\tilde{\lambda}_{M}^{(2)}$ of Ref. 26] is identical to our $K_{2}^{\text {mag }}$. The rotational-tidal perturbations appear in the $t, r r$ and $A B$ components of the metric. One can show that they all agree when the assignment

$$
\gamma_{32}^{*}=-\frac{192}{5} \sqrt{7 \pi} \mathfrak{K}^{\mathrm{o}} M^{2} \mathcal{B}_{0}^{\mathrm{q}}
$$

is made, based on the equivalence of our $\delta g_{t t}^{\ell=3}=k_{t t}^{o} \mathcal{K}^{\circ}=k_{t t}^{\mathrm{o}} \frac{3}{5} \chi \mathcal{B}_{0}^{\mathrm{q}} Y^{30}$ and $\delta g_{t t}^{\ell=3}=e^{\nu} \delta H_{0}^{(3)} Y^{30}$ of Ref. [26]. This association implies that the second mapping given in Eq. 25) relates the scaled gravitomagnetic rotational-tidal Love numbers.

By similar identifications, one can also match the dipole rotational-tidal perturbations, which contain gauge constants rather than Love numbers. Applying the relations derived above, it is straightforward to verify that the exterior metric of Ref. 26] is completely identical to Eq. 10 in axisymmetry. Ultimately, our Love number definitions differ only from those of Pani, Gualtieri and Ferrari by the purely conventional multiplicative factors which we have worked out here.

[1] B. P. Abbott et al., "GW170817: Observation of Gravitational Waves from a Binary Neutron Star Inspiral," Physical Review Letters 119, 161101 (2017).

[2] D. Lai, F. A. Rasio, and S. L. Shapiro, "Hydrodynamic instability and coalescence of binary neutron stars," Astrophys. J. 420, 811-829 (1994) astro-ph/9304027.

[3] C. S. Kochanek, "Coalescing binary neutron stars," Astrophys. J. 398, 234-247 (1992)

[4] É. É. Flanagan and T. Hinderer, "Constraining neutron star tidal Love numbers with gravitational wave detectors," Phys. Rev. D 77, 021502 (2008), arXiv:0709.1915 [astro-ph]

[5] T. Hinderer, "Tidal Love Numbers of Neutron Stars," Astrophys. J. 677, 1216-1220 (2008), arXiv:0711.2420

[6] M. Favata, "Are neutron stars crushed? Gravitomagnetic tidal fields as a mechanism for binary-induced collapse," Phys. Rev. D 73, 104005 (2006), astro-ph/0510668.

[7] T. Damour and A. Nagar, "Relativistic tidal properties of neutron stars," Phys. Rev. D 80, 084035 (2009), arXiv:0906.0096 $[\mathrm{gr}-\mathrm{qc}]$,

[8] T. Binnington and E. Poisson, "Relativistic theory of tidal Love numbers," Phys. Rev. D 80, 084018 (2009) arXiv:0906.1366 [gr-qc]

[9] P. Landry and E. Poisson, "Relativistic theory of surficial Love numbers," Phys. Rev. D 89, 124011 (2014), arXiv:1404.6798 $[\mathrm{gr}-\mathrm{qc}]$,

[10] P. Landry and E. Poisson, "Gravitomagnetic response of an irrotational body to an applied tidal field," Phys. Rev. D 91, 104026 (2015), arXiv:1504.06606 [gr-qc]

[11] X. H. Zhang, "Multipole expansions of the general-relativistic gravitational field of the external universe," Phys. Rev. D 34, 991-1004 (1986).

[12] J. S. Read, C. Markakis, M. Shibata, K. Uryū, J. D. E. Creighton, and J. L. Friedman, "Measuring the neutron star equation of state with gravitational wave observations," Phys. Rev. D 79, 124033 (2009), arXiv:0901.3258 [gr-qc].

[13] T. Hinderer, B. D. Lackey, R. N. Lang, and J. S. Read, "Tidal deformability of neutron stars with realistic equations of state and their gravitational wave signatures in binary inspiral," Phys. Rev. D 81, 123016 (2010), arXiv:0911.3535 [astro-ph.HE] 
[14] T. Damour, A. Nagar, and L. Villain, "Measurability of the tidal polarizability of neutron stars in late-inspiral gravitationalwave signals," Phys. Rev. D 85, 123007 (2012), arXiv:1203.4352 [gr-qc].

[15] J. S. Read, L. Baiotti, J. D. E. Creighton, J. L. Friedman, B. Giacomazzo, K. Kyutoku, C. Markakis, L. Rezzolla, M. Shibata, and K. Taniguchi, "Matter effects on binary neutron star waveforms," Phys. Rev. D 88, 044042 (2013) arXiv:1306.4065 [gr-qc]

[16] A. Maselli, L. Gualtieri, and V. Ferrari, "Constraining the equation of state of nuclear matter with gravitational wave observations: Tidal deformability and tidal disruption," Phys. Rev. D 88, 104040 (2013), arXiv:1310.5381 [gr-qc].

[17] S. Postnikov, M. Prakash, and J. M. Lattimer, "Tidal Love numbers of neutron and self-bound quark stars," Phys. Rev. D 82, 024016 (2010), arXiv:1004.5098 [astro-ph.SR]

[18] S. Y. Lau, P. T. Leung, and L.-M. Lin, "Tidal deformations of compact stars with crystalline quark matter," Phys. Rev. D 95, 101302 (2017), arXiv:1705.01710 [astro-ph.HE]

[19] L. Baiotti, T. Damour, B. Giacomazzo, A. Nagar, and L. Rezzolla, "Analytic Modeling of Tidal Effects in the Relativistic Inspiral of Binary Neutron Stars," Physical Review Letters 105, 261101 (2010), arXiv:1009.0521 [gr-qc].

[20] L. Baiotti, T. Damour, B. Giacomazzo, A. Nagar, and L. Rezzolla, "Accurate numerical simulations of inspiralling binary neutron stars and their comparison with effective-one-body analytical models," Phys. Rev. D 84, 024017 (2011), arXiv:1103.3874 [gr-qc].

[21] J. Vines, É. É. Flanagan, and T. Hinderer, "Post-1-Newtonian tidal effects in the gravitational waveform from binary inspirals," Phys. Rev. D 83, 084051 (2011), arXiv:1101.1673 [gr-qc]

[22] F. Pannarale, L. Rezzolla, F. Ohme, and J. S. Read, "Will black hole-neutron star binary inspirals tell us about the neutron star equation of state?" Phys. Rev. D 84, 104017 (2011), arXiv:1103.3526 [astro-ph.HE]

[23] B. D. Lackey, K. Kyutoku, M. Shibata, P. R. Brady, and J. L. Friedman, "Extracting equation of state parameters from black hole-neutron star mergers: Nonspinning black holes," Phys. Rev. D 85, 044061 (2012), arXiv:1109.3402 [astro-ph.HE],

[24] B. D. Lackey, K. Kyutoku, M. Shibata, P. R. Brady, and J. L. Friedman, "Extracting equation of state parameters from black hole-neutron star mergers: Aligned-spin black holes and a preliminary waveform model," Phys. Rev. D 89, 043009 (2014), arXiv:1303.6298 [gr-qc]

[25] M. Hannam, D. A. Brown, S. Fairhurst, C. L. Fryer, and I. W. Harry, "When can Gravitational-wave Observations Distinguish between Black Holes and Neutron Stars?" Astrophys. J. Lett. 766, L14 (2013), arXiv:1301.5616 [gr-qc]

[26] P. Pani, L. Gualtieri, and V. Ferrari, "Tidal Love numbers of a slowly spinning neutron star," Phys. Rev. D 92, 124003 (2015), arXiv:1509.02171 [gr-qc]

[27] P. Landry and E. Poisson, "Tidal deformation of a slowly rotating material body. External metric," Phys. Rev. D 91, 104018 (2015), arXiv:1503.07366 [gr-qc].

[28] P. Landry, "Tidal deformation of a slowly rotating material body: Interior metric and Love numbers," Phys. Rev. D 95, 124058 (2017), arXiv:1703.08168 [gr-qc]

[29] P. Landry and E. Poisson, "Dynamical response to a stationary tidal field," Phys. Rev. D 92, 124041 (2015), arXiv:1510.09170 [gr-qc].

[30] K. Yagi and N. Yunes, "I-Love-Q," Science 341, 365-368 (2013), arXiv:1302.4499 [gr-qc]

[31] E. Poisson, "Tidal deformation of a slowly rotating black hole," Phys. Rev. D 91, 044004 (2015), arXiv:1411.4711 [gr-qc].

[32] P. Pani, L. Gualtieri, A. Maselli, and Valeria Ferrari, "Tidal deformations of a spinning compact object," Phys. Rev. D 92, 024010 (2015), arXiv:1503.07365 [gr-qc]

[33] J. L. Synge, "Relativistic hydrodynamics," Proc. London Math. Soc. ser. 2, 376-400 43 (1938), 10.1112/plms/s2-43.5.376

[34] J. S. Read, B. D. Lackey, B. J. Owen, and J. L. Friedman, "Constraints on a phenomenologically parameterized neutronstar equation of state," Phys. Rev. D 79, 124032 (2009), arXiv:0812.2163 [astro-ph]

[35] P. Demorest, T. Pennucci, S. Ransom, M. Roberts, and J. Hessels, "A two-solar-mass neutron star measured using Shapiro delay," Nature 467, 1081-1083 (2010), arXiv:1010.5788 [astro-ph.HE].

[36] J. Antoniadis et al., "A Massive Pulsar in a Compact Relativistic Binary," Science 340, 1233232 (2013), arXiv:1304.6875 [astro-ph.HE]

[37] K. Yagi, L. C. Stein, G. Pappas, N. Yunes, and T. A. Apostolatos, "Why I-Love-Q: Explaining why universality emerges in compact objects," Phys. Rev. D 90, 063010 (2014), arXiv:1406.7587 [gr-qc]

[38] K. Yagi and N. Yunes, "I-Love-Q relations in neutron stars and their applications to astrophysics, gravitational waves and fundamental physics," Phys. Rev. D 88, 023009 (2013), arXiv:1303.1528 [gr-qc]

[39] K. Yagi and N. Yunes, "Approximate universal relations for neutron stars and quark stars," Physics Reports 681, 1-72 (2017), arXiv:1608.02582 [gr-qc]

[40] K. Yagi, "Multipole Love relations," Phys. Rev. D 89, 043011 (2014), arXiv:1311.0872 [gr-qc]

[41] T. Delsate, "I-Love relations for irrotational stars," Phys. Rev. D 92, 124001 (2015), arXiv:1504.07335 [gr-qc]

[42] K. Yagi and N. Yunes, "Binary Love relations," Class. Quantum Grav. 33, 13LT01 (2016), arXiv:1512.02639 [gr-qc].

[43] K. Yagi and N. Yunes, "Approximate universal relations among tidal parameters for neutron star binaries," Class. Quantum Grav. 34, 015006 (2017), arXiv:1608.06187 [gr-qc]

[44] E. Poisson and I. Vlasov, "Geometry and dynamics of a tidally deformed black hole," Phys. Rev. D 81, 024029 (2010), arXiv:0910.4311 [gr-qc].

[45] M. Mathisson, "Republication of: New mechanics of material systems," General Relativity and Gravitation 42, 1011-1048 $(2010)$

[46] A. Papapetrou, "Spinning Test-Particles in General Relativity. I," Proc. Royal Soc. London A 209, 248-258 (1951).

[47] E. Corinaldesi and A. Papapetrou, "Spinning Test-Particles in General Relativity. II," Proc. Royal Soc. London A 209, 
$259-268(1951)$

[48] J. L. Friedman and N. Stergioulas, Rotating Relativistic Stars (Cambridge University Press, 2013).

[49] B. Preston and E. Poisson, "A light-cone gauge for black-hole perturbation theory," Phys. Rev. D 74, 064010 (2006) arXiv:gr-qc/0606094 [gr-qc],

[50] F. Douchin and P. Haensel, "A unified equation of state of dense matter and neutron star structure," Astron. Astrophys. 380, 151 (2001), arXiv:astro-ph/0111092 [astro-ph].

[51] L. Engvik, E. Osnes, M. Hjorth-Jensen, G. Bao, and E. Ostgaard, "Asymmetric Nuclear Matter and Neutron Star Properties," Astrophys. J. 469, 794 (1996), nucl-th/9509016

[52] H. Müther, M. Prakash, and T.L. Ainsworth, "The nuclear symmetry energy in relativistic Brueckner-Hartree-Fock calculations," Phys. Lett. B 199, 469 - 474 (1987).

[53] H. Müller and B. D. Serot, "Relativistic mean-field theory and the high-density nuclear equation of state," Nucl. Phys. A 606, $508-537(1996)$

[54] B. D. Lackey, M. Nayyar, and B. J. Owen, "Observational constraints on hyperons in neutron stars," Phys. Rev. D 73, 024021 (2006), astro-ph/0507312.

[55] M. Alford, M. Braby, M. W. Paris, and S. Reddy, "Hybrid stars that masquerade as neutron stars," Astrophys. J. 629, 969-978 (2005), arXiv:nucl-th/0411016 [nucl-th]

[56] F. Özel, D. Psaltis, R. Narayan, and A. Santos Villarreal, "On the mass distribution and birth masses of neutron stars," Astrophys. J. 757, 55 (2012), arXiv:1201.1006 [astro-ph.HE].

[57] J. G. Martinez, K. Stovall, P. C. C. Freire, J. S. Deneva, F. A. Jenet, M. A. McLaughlin, M. Bagchi, S. D. Bates, and A. Ridolfi, "Pulsar J0453+1559: A Double Neutron Star System with a Large Mass Asymmetry," Astrophys. J. 812, 143 (2015), arXiv:1509.08805 [astro-ph.HE]

[58] N. Chamel and P. Haensel, "Physics of Neutron Star Crusts," Living Rev. Rel. 11, 10 (2008), arXiv:0812.3955 [astro-ph]

[59] L. C. Stein, K. Yagi, and N. Yunes, "Three-Hair Relations for Rotating Stars: Nonrelativistic Limit," Astrophys. J. 788, 15 (2014), arXiv:1312.4532 [gr-qc].

[60] E. Poisson and C. Will, Gravity (Cambridge University Press, 2014).

[61] E. Poisson and J. Douçot, "Gravitomagnetic tidal currents in rotating neutron stars," Phys. Rev. D 95, 044023 (2017) arXiv: 1612.04255 [gr-qc]

[62] C. E. Rhoades and R. Ruffini, "Maximum mass of a neutron star," Phys. Rev. Lett. 32, 324-327 (1974)

[63] E. D. Van Oeveren and J. L. Friedman, "Upper limit set by causality on the tidal deformability of a neutron star," Phys. Rev. D 95, 083014 (2017), arXiv:1701.03797 [gr-qc]

[64] H. O. Silva and N. Yunes, "I-Love-Q to the extreme," (2017), arXiv:1710.00919 [gr-qc]

[65] R. Price and K. S. Thorne, "Non-Radial Pulsation of General-Relativistic Stellar Models. II. Properties of the Gravitational Waves," Astrophys. J. 155, 163 (1969)

[66] A. Campolattaro and K. S. Thorne, "Nonradial Pulsation of General-Relativistic Stellar Models. V. Analytic Analysis for $\mathrm{L}=1, "$ Astrophys. J. 159, 847 (1970). 